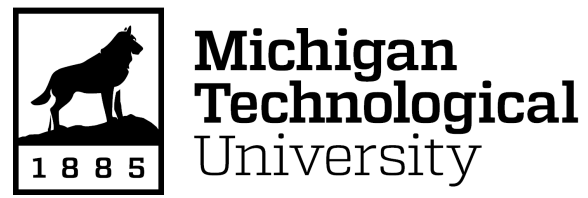

Michigan Technological University Digital Commons @ Michigan Tech

Dissertations, Master's Theses and Master's Reports

2017

\title{
INVESTIGATING MIRNA FUNCTIONS IN ARABIDOPSIS BY SHORT TANDEM TARGET MIMIC (STTM)
}

Yiyou Gu

Michigan Technological University, yiyoug@mtu.edu

Copyright 2017 Yiyou Gu

\section{Recommended Citation}

Gu, Yiyou, "INVESTIGATING MIRNA FUNCTIONS IN ARABIDOPSIS BY SHORT TANDEM TARGET MIMIC (STTM)", Open Access Dissertation, Michigan Technological University, 2017.

https://doi.org/10.37099/mtu.dc.etdr/430

Follow this and additional works at: https://digitalcommons.mtu.edu/etdr

Part of the Biology Commons, Developmental Biology Commons, and the Molecular Genetics Commons 


\title{
INVESTIGATING MIRNA FUNCTIONS IN ARABIDOPSIS BY SHORT TANDEM TARGET MIMIC (STTM)
}

\author{
By \\ Yiyou Gu
}

A DISSERTATION

Submitted in partial fulfillment of the requirements for the degree of DOCTOR OF PHILOSOPHY

In Biological Sciences

MICHIGAN TECHNOLOGICAL UNIVERSITY

2017

(C) 2017 Yiyou $\mathrm{Gu}$ 
This dissertation has been approved in partial fulfillment of the requirements for the Degree of DOCTOR OF PHILOSOPHY in Biological Sciences.

Department of Biological Sciences

Dissertation Advisor: $\quad$ Dr. Guiliang Tang

Committee Member: Dr. Xiaoqing Tang

Committee Member: Dr. Haiying Liu

Committee Member: Dr. Michael Gretz

Department Chair: Dr. Chandrashekhar P. Joshi 


\section{Table of Contents}

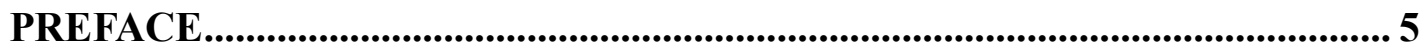

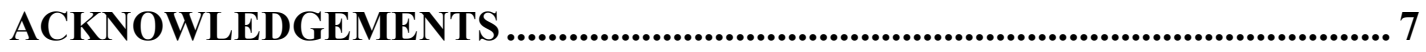

ABSTRACT …........................................................................................................... 9

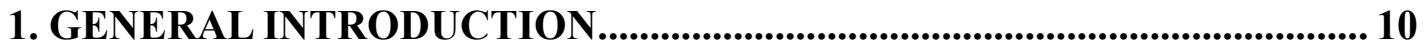

1.1. THE APPROACH TO MIRNA FUNCTION IN ARABIDOPSIS.................................. 10

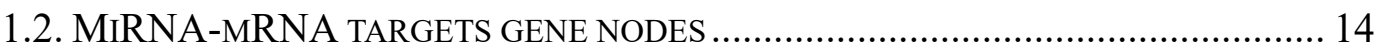

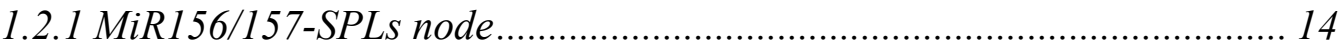

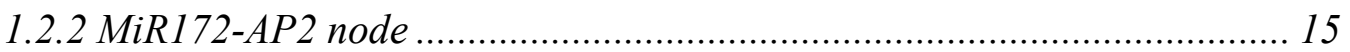

1.2.3 MiR390- TAS3 tasiRNA-ARF3 \& 4 node .............................................. 16

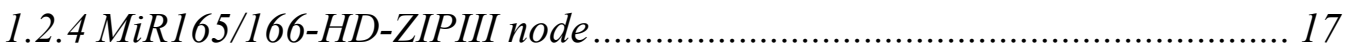

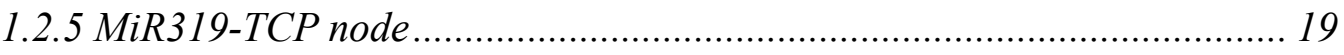

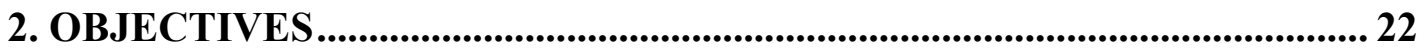

3. BLOCKING MIRNAS IN ARABIDOPSIS BY SHORT TANDEM TARGET

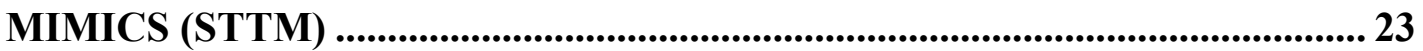

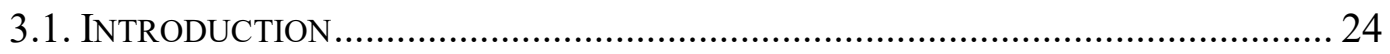

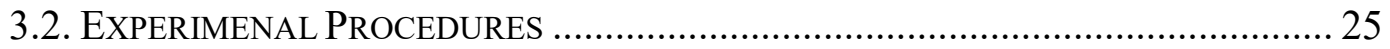

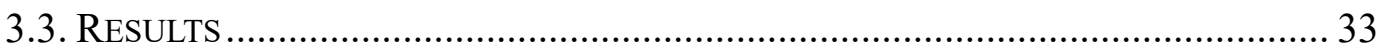

3.3.1 Blocking miR165/166 activity by STTM transcript in vivo....................... 33

3.3.2 The spacer length between two miRNA binding sites is important .......... 38

3.3.3 STTM165/166-48nt transcripts induced miR165/166 degradation........... 44

3.3.4 Blocking other miRNAs in Arabidopsis by STTM .................................. 49

3.3.5 SDNs play an important role in STTM induced miRNA degradation....... 53

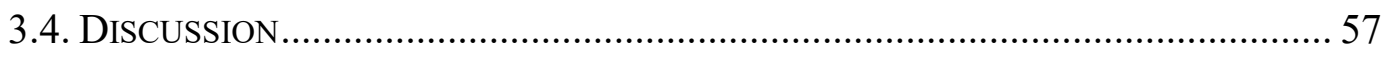

4. MIRNAS CAN REGULATE PLANT ANTHOCYANIN ACCUMULATION

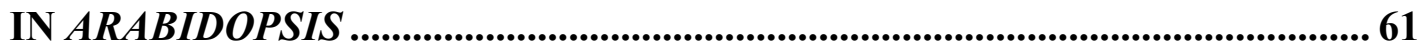

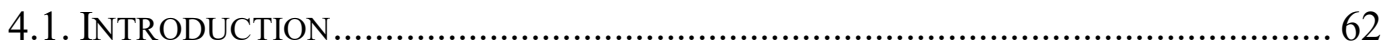

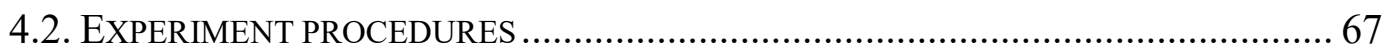

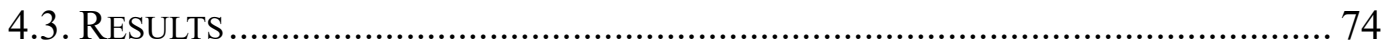

4.3.1 Functional blockage of both miR165/166 and miR156/157 by crossing between STTM165/166-48nt and STTM156/157.......................................... 74

4.3.2 MiR165/166 and miR156/157 were simultaneously and functionally

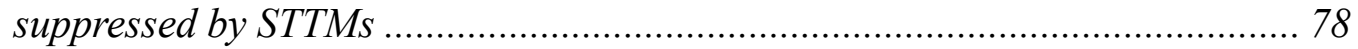

4.3.3 MiR165/166 regulates anthocyanin predominantly in leaves, while 
miR156/157 regulates anthocyanin in both leaves and stems ....................... 81

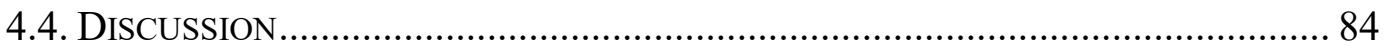

\section{INVESTIGATING MIR165/166 REGULATORY NETWORK TO IN}

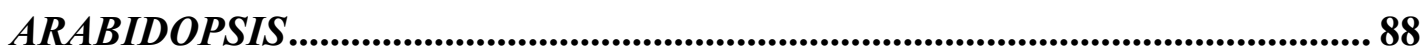

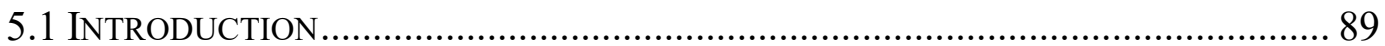

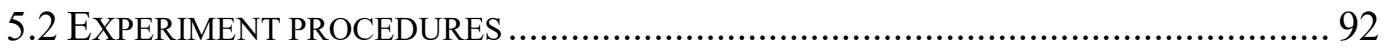

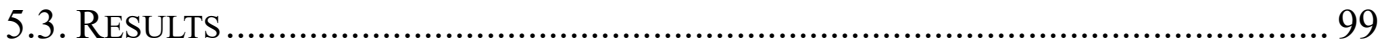

5.3.1 Repression of miR165/166 induce the disordered expression of other

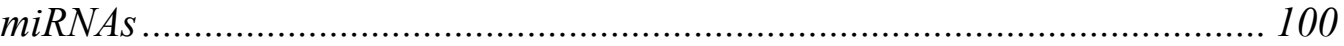

5.3.2 phb-phv-cna promoting miR319 expression and repressing REV

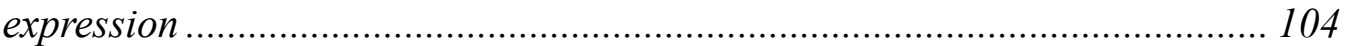

5.3.3 MiR319 expression in HD-ZIP III single genetic mutant plants ............ 106

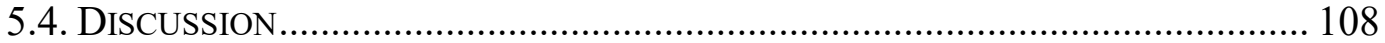

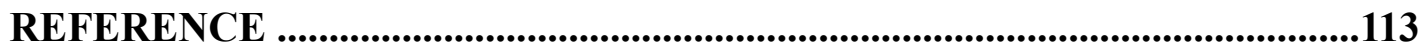

APPENDIX …............................................................................................................. 121 


\section{Preface}

My PhD dissertation explores the function of miR165/166 and the regulatory network between miR165/166 and other individual miRNAs. The findings of my research are composed of three chapters: Chapter 3 to 5 .

Chapter 3. Blocking miRNA in Arabidopsis by short tandem target mimics (STTM).

The partial of this chapter is published in the Journal of Plant Cell with me as the co-first author.

(Yan, J., Y. Gu, X. Jia, W. Kang, S. Pan, X. Tang, X. Chen and G. Tang (2012). "Effective small RNA destruction by the expression of a short tandem target mimic in Arabidopsis." Plant Cell 24(2): 415-427).

Chapter 4. MiRNAs can regulate plant anthocyanin accumulation in Arabidopsis 
This chapter is planned to be included for research article (manuscript under preparation). Guiliang Tang and I are writing the manuscript, I designed, performed and collected the results of the experiments

Chapter 5. Exploring potential miRNAs that have a regulatory network with miR165/166

This chapter will be a part of the research article (manuscript under preparation) that we plan to submit in the future. Guiliang Tang and I are writing the manuscript. I designed, performed and collected the results of the experiments. 


\section{Acknowledgements}

I would like to thank my advisor, Dr. Guiliang Tang for giving me the opportunity to work with him. I would like to thank his support that helped me achieve my research goals. I would also like to thank the Department of Biological Sciences for supporting and giving me such a wonderful environment to work.

I would like to thank Dr. Xiaoqing Tang, Dr. Michael R. Gretz, and Dr. Haiying Liu for being in my advisory committee and guiding me throughout my degree program with their inspiring scientific thinking and ideas. I have always been motivated to achieve more by seeking their advice.

I also thank my colleagues Haiping Liu, Yiping Mao, Mengmeng Qiao, Li Chen, Lei Tian, Lina Shi, Sachin Teotia for their friendship and willingness to help me in doing experiments and lab duties. My sincere gratitude for Dr. Chandrashekhar P. Joshi, Dr. Nancy Auer, Dr. Rupali Datta, 
Patricia A. Asselin, Jeffrey Lewin and Emily Betterly. Their kindly help made me focused more on my research.

Also thanks to all my friends from Michigan Tech University. Their presence around me means a lot to me. 


\section{Abstract}

MicroRNA (miRNAs) and other endogenous small RNAs play important role in regulation gene expression in eukaryotes. Till now, several techniques have been developed for miRNA function analysis. Because of the characteristics of miRNA families normally with multiple members, the most applicable method for miRNA function research is to block the whole miRNA family specifically. Here, we report an artificial non-coding transcript termed Short Tandem Target Mimic (STTM) for blocking miRNA activity in Arabidopsis thaliana. The STTM composed of two miRNA target sites with a three-nucleotide bulge in the miRNA cleavage site, the two-miRNA target sites separated by spacer linker. We used this technique to block several important endogenous miRNA with high efficiency; we also demonstrated that the degradation of miRNA induced by small RNA degradation nucleases (SDNs). Furthermore, we applied STTM to study the regulatory network and functional interactions of a few miRNAs in Arabidopsis. In summary, we generated an 
effective approach to block endogenous miRNA and used it to study a number of miRNAs in Arabidopsis, therefor providing a powerful tool for miRNA function research in plants.

\section{General Introduction}

\subsection{The approach to miRNA function in Arabidopsis}

Small RNAs, including microRNAs (miRNAs) and small interfering RNAs (siRNAs), play very important roles in growth and development, epigenetics, genome integrity, defense against viral infection, and response to environmental changes in plants [1]. MiRNAs are especially important in controlling plant development, since most miRNA target genes in plants are transcription factors; these transcription factors regulate plant development and phase change in different growing stages. The major role of short interfering RNAs (siRNAs) is to regulate mRNA stability post-transcriptionally and genome-wide histone methylation [2]. To date, hundreds of 
miRNAs and thousands of endogenous siRNAs have been identified from many plant species, and dissection of their functions will help us better understand their regulatory mechanism [3]. Such functional dissection of individual small RNAs in vivo requires an effective means to block their production or activity.

Traditional approaches to the interrogation of gene function rely on the generation and characterization of genetic mutants. Such genetic approaches are not applicable to the investigation of small RNA functions due to their small size and the fact that many miRNA families are composed of multiple members with potentially overlapping functions. It is almost impossible to obtain mutations in all the miRNA family members in a single plant by a traditional approach. Another indirect approach to miRNA functions is to build miRNA-resistant target lines, which silently mutate in the miRNA binding site on the target mRNA without changing the translated protein sequence. Such an approach is effective to investigate miRNA families with just a single target [4]. However, given that a 
miRNA usually regulates multiple target genes with either redundant or distinct functions, this approach could only partially reveal the functions of a particular miRNA in vivo.

The latest approach is using one genetic transformation to block all the members of one miRNA family and thus de-repress the miRNA-repressed targets. The working mechanism underlying such an approach was first discovered in Arabidopsis as target mimicry (TM) from the interaction between miR399 and INDUCED BY PHOSPHATE STAVATION1 (IPS1) [5]. MiR399 is induced by phosphate starvation in Arabidopsis [6]. The target gene of miR399 is E2 conjugating enzyme UBC24 (PHO2); the function of $\mathrm{PHO} 2$ is to enhance phosphorus acquisition in plants[7]. At the same time under phosphate starvation, the transcripts of the non-protein-coding gene, IPS1, are induced[8]. IPS1 transcripts are partially complementary to miR399, forming a bulge in the central region of the miR399 binding site, when pairing with miR399. Unlike PHO2 that is cleaved by miR399, 
IPS1 is functionally sequestering miR399 than being cleaved by miR399 [9].

The TM approach was recently further developed into a powerful technology termed short tandem target mimic (STTM), which has been successfully applied to other model and crop plants, including rice, maize, tobacco, and tomato[10-12]. In the first chapter of this study, I will present how this STTM technology was developed during my early studies. We blocked several important miRNAs including miR165/166, miR156/156, and miR319 in Arabidopsis using STTM technique. The miR165/166 targets are homeodomain-leucine zipper III (HD-ZIP III) family genes, functional on formation of Shoot apical meristem (SAM)[13]. MiR156/157 target gene is SQUAMOSA PROMOTER BINDING PROTEINLIKE (SPLs) family genes, mainly working on the plant phase transition and plant flowering timing[14]. MiR319 target TCP controls many aspects of plant growth and especially leaf development[15]. 


\subsection{MiRNA-mRNA target gene nodes}

Plant miRNAs are usually complementary with their mRNA targets sequence extensively, while most animal miRNAs interact with their targets through the limited "seed" regions forming extensive mismatches between the miRNAs and their targets. Thus, in animals, one miRNA may target hundreds of genes, but plant miRNA has a limited number of targets $[16$, 17].

The relationship between each specific miRNA or miRNA family and its target genes can be defined as a miRNA node. There are many reported miRNA-target nodes[18] revealed by previous research. The regulation of miRNA nodes is extremely dynamic, subject to temporal and spatial changes during different stages of plant development. The following miRNA nodes have been well defined.

\subsubsection{MiR156/157-SPLs node}


MiR156/157 have been revealed to play a key role in the control of plant phase change and flower timing. SPLs can be divided into two main classes: the SPL3-SPL4-SPL5 class and the SPL9-SPL15 class[19]. miR156 expression level is high at the young stage and then decreases as the plant develops into adult and later reproduction stages. miR156 expression levels reach the lowest level at the flowering stage, resulting in an elevation in the expressions of the target genes of SPL3-SPL4-SPL5 class that activate flower-promoting MADS box genes[20-22]. In contrast, the SPL9-SPL15 class has a strong effect on leaf initiation rate and the trichome formation. SPL9 expression in shoot apices increases when plants grow from the vegetative phase to flowering phase[23].

\subsubsection{MiR172-AP2 node}

In the miR172-AP2 node, miR172 regulates the plant-specific transcription factor gene Apetala 2 (AP2) and a small group of AP2-like genes, such as TARGET OF EAT1 (TOE1), TOE2, 
and TOE3[20, 24]. In contrast to the miR156/157-SPL node, the miR172 expression level is up regulated when plants grow from the adult stage to the flowering stage. As a result, the expression of miR172 target genes decrease. AP2 expresses specifically in floral organs to regulate flowering and flower development $[25,26]$. Moreover, at least five AP2-like genes function as flowering repressors in Arabidopsis. Actually, miR172-AP2 node interacts with the miR156/157-SPL node to regulate plant developmental stages during the juvenile to adult transition.

\subsubsection{MiR390- TAS3 tasiRNA-ARF3 \& 4 node}

The MiR390-tasiRNA-TAS3 node is also involved in plant phase transition. In this node, miR390 initiates the production of trans-acting siRNA (tasiRNA), from the TAS3 locus through the so-called tasiRNA pathway, composed of RNA-dependent RNA polymerase 6 (RdR6), Dicer-like 4 DCL4, and AGO7[27]. The target genes of the tasiRNA are AUXIN 
RESPONSE FACTOR 3 (ARF3) and ARF4, which promote plant development into adult phase [28]. In the plant with deficient TAS3 or with Argonaute 7 (ago7) mutation, a higher expression level of ARF3 and ARF4 gene were detected[29].

\subsubsection{MiR165/166-HD-ZIPIII node}

The MiR165/166-HD-ZIPIII node is one of the most important and well-studied miRNA nodes so far studied. MiR165 and 166 (miR165/166) are one of the most abundant miRNA families that are expressed in plants. MiR165 and miR166 have almost identical sequence except for the 17th nucleotide[30]. There are seven copies of the miR166 gene and two copies of the miR165 gene in the Arabidopsis Genus[31]. Both miR165 and miR166 target the Class III HOMEODOMAIN-LEUCINE ZIPPER transcriptional factor (HD-ZIP III) family gene transcripts for cleavage through the base paring between miR165/166 and HD-ZIPIII mRNAs. The HD-ZIP III family contains five genes: PHABULOSA 
(PHB), PHAVOLUTA (PHV), REVOLUTA (REV), ATHB8 and ATHB15[30]. The HD-ZIP III genes encode plant-specific transcription factors that are involved in many aspects of plant development, including the specification of abaxial-adaxial identity in leaf and vascular development[32]. Abnormal expression of HD-zip III family genes leads to the loss of leaf and vascular asymmetry due to a disturbed change in abaxial-adaxial patterning.

Both down- and up-regulation of miR165/166 affect the expressions of their target genes and further plant development. When the miR165/166 is repressed by a short tandem target mimic (STTM165/166) structure in Arabidopsis, the expression of miR165/166 target genes of the HD-zip III family is up regulated several fold compared with the wild-type plant. STTM165/166 plants displayed dramatic phenotypic alterations in development, including twisted leaf and stem [33]. On the other hand, overexpression of miR166 by activating tagging results in down-regulation of the PHV, PHB and ATHB-15 genes. Similarly, transgenic plants 
over-expressing miR165/166 displayed a loss of shoot apical meristems (SAMs) and an enhancement in vascular development[34].

miR165/166 are not unique in regulating the HD-ZIP III family through direct targets cleavage, another kind of small ZPR proteins also affect the expression of HD-ZIPIII genes through physical interactions with HD-ZIPIII proteins[35]. For example, ZIP3 and its redundant homolog, ZIP4, negatively regulate the HD-ZIP III activity in SAM development [36]. The zpr3-1d mutant, which was isolated from the Arabidopsis activation-tagged mutant pool, was phenotypically similar to the miR165/166 overexpressed mutant plant [36]. ZPR, with a gain-of-function mutation, can repress the HD-ZIP III gene activity to a level similar to miR165/166 directed down-regulation of HD-ZIP mRNAs.

\subsubsection{MiR319-TCP node}


miR319 was discovered by activating tagging screening and MiR319 target is TCP transcription factor [37]. In activating tagging screening, a T-DNA containing a $35 \mathrm{~S}$ enhancer is integrated randomly into Arabidopsis genome. There has been one mutant identified for its unusual morphology, and this mutant line was termed jaw-D, based on the observation that the plant leaves appeared jagged and crinkled[38]. Soon after, jaw-D was identified to be miR319. Microarray experiments demonstrated that TCP family genes were significantly down regulated in the jaw-D mutant. TCP family genes were finally identified to be the direct target genes of miR319, based on the fact that the TCP family genes shared a highly conserved complementary region to the miR319. The TCPs belong to a plant-specific transcription factor family that regulates downstream gene expression for cellular activities including leaf cell division, expansion and differentiation during the plant development $[15,39]$. In Arabidopsis, the TCP family contains 24 members that can be divided into two main groups (class I and II) according to the variations of their sequences [40]. Among the 24 TCP family genes, 5 members, TCP2, 
TCP3, TCP4, TCP10, and TCP24, contain a target site for miR319, and all these 5 TCPs belong to the class II group[41]. 


\section{Objectives}

This dissertation has the following three objectives.

Objective 1: Generate a new short non-coding transcript to block miRNA function in Arabidopsis

Objective 2: Understand the role of miRNAs in anthocyanin accumulation in Arabidopsis

Objective 3: Determine the miRNA regulatory network related to miR165/166 in Arabidopsis 


\section{Blocking miRNAs in Arabidopsis by short tandem target mimics (STTM)}

Jun Yan, Yiyou Gu, Xiaoyun Jia, Wenjun Kang, Shangjin Pan, Xiaoqing

Tang, Xuemei Chen, Guiliang Tang

The partial data of this chapter is published in the Journal of Plant Cell. 


\subsection{Introduction}

MicroRNAs (miRNAs) are a class of endogenous small RNAs that regulate gene expressions post-transcriptionally. MiRNAs are 20-23 nt in length, and interact with the protein AGOs, forming RNA-induced silencing complexes (RISCs) to cleave their target gene transcripts or repress the translation of the target mRNAs [42]. The miRNAs are widely functioning in plant developmental processes due to that most miRNA targets belong to all kinds of transcription factors, which control plant phase change, DNA methylation, chromatin remodeling, plant immunity, and responses to biotic and abiotic stresses [43-45]. Hundreds of miRNAs have been identified in plants [46], but their functions have been only partially dissected using traditional approaches. An endogenous non-coding transcript IPS1 modulates miR399 activity, termed target mimicry (TM), was reported in Arabidopsis[6]. MiR399 is induced by phosphate starvation and regulates its target PHO2. The non-coding transcript IPS1 is also induced by phosphate 
starvation. The IPS1 is partially complementary to miR399, leading to miR399 is functional sequestrated by IPS1, but miR399 can not cleavage the IPS1 transcripts due to the 3 nucleotides exist in the central of miR399 target sequence in IPS1 transcript[6]. Through replacing miR399 target sequence with other miRNA sequence, a large-scale study based on TM for miRNA function research has been completed, but the results indicate that only partial miRNAs were effectively blocked by TM [5]. To generated a TM-like transcript which can block miRNA activity more efficiently, we designed a transcript with two partial complementary miRNA target sites, similar to IPS1 based TM, and a 48nt nucleotides spacer was added between the two miRNA target sites to stabilize the structure. In this chapter, I mainly introduce how I developed the technology termed short tandem target mimic (STTM) and used it to dissect the functions of miRNAs in Arabidopsis.

\subsection{Experimental Procedures}




\section{Construction of the STTM plasmid and transgenes}

The STTM module was assembled in the pOT2 vector between a 2 X35S promoter and a $35 \mathrm{~S}$ terminator by PCR amplification. The STTM structure was amplified, together with the pOT2 vector, by back-to-back PCR using two long primers containing a SwaI site at the 5' end of each primer. The back-to-back PCR product containing the pOT2 backbone with the chloramphenicol selection marker was purified using a PCR product purification kit (Promega) and then digested by SwaI restriction endonuclease. The digested PCR product was further purified by PCR purification as above and self-ligated overnight at room temperature. The ligated product was transformed into XL1-blue competent cells. We selected single colonies for plasmid purification. The recombinant constructs were verified by linearization of the plasmids after SwaI digestion [47], and the non-recombinant plasmids that could not be linearized by SwaI were abandoned. Next, a pair of primers that contain PacI site at the 5' end of each primer were 
used to amplify the recombinant plasmid to delete the origin of the plasmid. The PCR product containing the STTM structure and pOT2 backbone without the plasmid replication origin was purified and digested by SwaI, to be cloned into a modified binary vector PFGC5941 containing a Kanamycin selection marker through a unique SwaI. The SwaI digested products of the PCR fragment and PFGC5941 were purified and ligated to each other overnight with T4 DNA ligase (NEB Inc.). The ligated product was transformed into XL1-blue competent cells, and the recombinant plasmids, which contain both binary vector PFGC5941, and the PCR product were selected on LB plates containing Chloramphenicol and Kanamycin. The recombinant plasmids were isolated for further identification by linearization of the plasmids using SwaI and DNA sequencing. The verified plasmids were used for plant transformation.

To investigate the efficiencies of STTMs containing either one or two miRNA binding sites, we designed two modified primers that contain the STTM structure with mutations on 
either miRNA binding site to generate the STTM mutants, STTM165mut/166 and STTM165/166mut, for plant transformation. The primers used for STTM constructs are listed in Table 1.

Transgenic plants were generated by Agrobacterium tumefaciens-mediated floral dip transformation[48]. The seeds were collected after floral dip; transgenic plants with resistance to the herbicide BASTA will be screened for further analysis. 
Table 1: The primers for STTM constructs in this study.

\begin{tabular}{|c|c|c|}
\hline Construct & Primer name & Sequences \\
\hline \multirow[b]{2}{*}{ STTM165/166-8 } & 165-166-STTMSwa8ntlink-PF & $\begin{array}{l}\text { GCCATTTAAATGGGGAATGAAGCTACCTGGTCCGAGAATTCGGTACGCTGAAAT } \\
\text { CACCAG }\end{array}$ \\
\hline & 165-166-STTMSwa8ntlink-PR & $\begin{array}{l}\text { GCCATTTAAATTCGGACCAGGTAGCTTCATCCCCCAAGCTTGGGCTGTCCTCTCC } \\
\text { AAATG }\end{array}$ \\
\hline \multirow[b]{2}{*}{ STTM165/166-31 } & 165-166-STTMSwa31ntlink-PF & $\begin{array}{l}\text { GCCATTTAAATATGGTCTAGGGGAATGAAGCTACCTGGTCCGAGAATTCGGTAC } \\
\text { GCTGAAATCACCAG }\end{array}$ \\
\hline & 165-166-STTMSwa31ntlink-PR & $\begin{array}{l}\text { GCCATTTAAAGGCAAATTAGACCATTCGGACCAGGTAGCTTCATCCCCCAAGCT } \\
\text { TGGGCTGTCCTCTCCAAATG }\end{array}$ \\
\hline \multirow[b]{2}{*}{ STTM165/166-48 } & 165-166-STTMSwa48ntlink-PF & $\begin{array}{l}\text { GCCATTTAAATATGGTCTAAAGAAGAAGAATGGGGAATGAAGCTACCTGGTCCG } \\
\text { AGAATTCGGTACGCTGAAATCACCAG }\end{array}$ \\
\hline & 165-166-STTMSwa48ntlink-PR & $\begin{array}{l}\text { GCCATTTAAATTAGACCATAACAACAACAACTCGGACCAGGTAGCTTCATCCCC } \\
\text { CAAGCTTGGGCTGTCCTCTCCAAATG }\end{array}$ \\
\hline \multirow[b]{2}{*}{ STTM165/166-88 } & 165-166-STTMSwa88ntlink-PF & $\begin{array}{l}\text { GCCATTTAAATATGGTCTAAAGAAGAAGAATATGGTCTAAAGAAGAAGAATGGG } \\
\text { GAATGAAGCTACCTGGTCCGAGAATTCGGTACGCTGAAATCACCAG }\end{array}$ \\
\hline & 165-166-STTMSwa88ntlink-PR & $\begin{array}{l}\text { GCCATTTAAATTAGACCATAACAACAACAACTAGACCATAACAACAACAACTCG } \\
\text { GACCAGGTAGCTTCATCCCCCAAGCTTGGGCTGTCCTCTCCAAATG }\end{array}$ \\
\hline \multirow[b]{2}{*}{ STTM165/166-96 } & 165-165-STTMSwa96ntlink-PF & $\begin{array}{l}\text { GCCATTTAAATATGGTCTAAAGAAGAAGAATATGGTCTAAAGAAGAAGAATATG } \\
\text { GGGGGAATGAAGCTACCTGGTCCGAGAATTCGGTACGCTGAAATCACCAG }\end{array}$ \\
\hline & -STTMSwa96ntlink-PR & $\begin{array}{l}\text { GCCATTTAAATTAGACCATAACAACAACAACTAGACCATAACAACAACAACTAG } \\
\text { ATCGGACCAGGTAGCTTCATCCCCCAAGCTTGGGCTGTCCTCTCCAAATG }\end{array}$ \\
\hline \multirow{2}{*}{$\begin{array}{c}\text { STTM165del/ } \\
166-48\end{array}$} & 165 del-166-STTMSwa 48 ntllink-PF & $\begin{array}{l}\text { GCCATTTAAATATGGTCTAAAGAAGAAGAATGGGGAATGAAGCTACCTGGTCCG } \\
\text { AGAATTCGGTACGCTGAAATCACCAG }\end{array}$ \\
\hline & 165del-166-STTMSwa48ntlink-PR & GCCATTTAAATAAGCTTGGGCTGTCCTCTCCAAATG \\
\hline \multirow[b]{2}{*}{ STTM156/157-48 } & 156-157-STTMSwa48ntlink-PF & $\begin{array}{l}\text { GCCATTTAAATATGGTCTAAAGAAGAAGAATGTGCTCTCTATCTACTTCTGTCAA } \\
\text { GAATTCGGTACGCTGAAATCACCAG }\end{array}$ \\
\hline & 156-157-STTMSwa48ntlink-PR & $\begin{array}{l}\text { GCCATTTAAATTAGACCATAACAACAACAACTGACAGAAGATAGGAGTGAGCA } \\
\text { CTAAGCTTGGGCTGTCCTCTCCAAATG }\end{array}$ \\
\hline \multirow[b]{2}{*}{ STTM319-48 } & 319-STTMSwa8ntlink-PF & $\begin{array}{l}\text { GCC ATT TAAATA TGG TCT AAA GAA GAA GAA TAG GGA GCT CCC CTA TTC } \\
\text { AGT CCA AGA ATT CGG TAC GCT GAA ATC ACC AG }\end{array}$ \\
\hline & 319-STTMSwa8ntlink-PF & $\begin{array}{l}\text { GCC ATT TAAATT AGA CCA TAA CAA CAA CAA CTT GGA CTG AAT AGG GGA } \\
\text { GCT CCC TAA GCT TGG GCT GTC CTC TCC AAA TG }\end{array}$ \\
\hline \multirow[b]{2}{*}{ Origin Deletion } & Origin-del-Pacl-PF & TCCCTTAATTAAGTTTGCAAGCAGCAGATTACGCG \\
\hline & Origin-del-Pacl-PR & TCCCTTAATTAAGAAAGGCGGACAGGTATCCGGTAAG \\
\hline
\end{tabular}

\section{Small RNA gel blotting}

Total RNA was isolated from leaves of Arabidopsis transgenic and control plants of $\mathrm{T} 1$ or later generations using trizol (Invitrogen), and 10 ug of total RNA was resolved by $15 \%$ PAGE under denaturing conditions and transferred onto blots. $\mathrm{P}^{32}$ labeled probes complementary to the small RNA sequence were used for hybridization with blots [49]. To detect STTM transcripts in transgenic plants by Northern blotting, the STTM 
reverse primers were labeled with $\mathrm{P}^{32}$ as probes. U6 expression was detected as an internal control.

\section{Detecting mRNA expression by qRT-PCR}

After the transgenic plants were screened and validated for the STTM-triggered degradation of miRNA by Northern blot confirmation, the expression of the miRNA target genes was detected by qRT-PCR. Total RNA was isolated from leaves using RNeasy mini kit (Qiagen). DNase was used to treat the purified total RNA to remove DNA contamination before the RNA reverse transcription. The random primer was used as the reverse transcription primer. qRT-PCR using the SYBR green PCR master mix kit was performed with an Applied Biosystems step one instrument (ABI). Actin mRNA was used as an internal control. The relative levels of gene expression were calculated using $2-^{\wedge}$ cycle threshold method [50]. Three biological replicates were examined to ensure reproducibility. 


\section{Detecting the ability of STTM165/166-48nt transcripts in sequestering miR165/166 in vitro}

We generated STTM165/166-48nt RNAs in vitro using T7 RNA polymerase. The DNA templates were made by the PCR amplification. The primers used for the PCR were: T7-STTM165/166-48-PF and T7-STTM165/166-48-PR. The STTM-Spacer-48nt-template was used for STTM165/166-48nt in vitro transcription. The transcription method was described previously[51]. The miR165/166 was synthesized (Thermo

Fisher Scientific). Synthesized MiR165/166 and STTM165/166 transcripts were labeled at 5' end [52]. The labeled miR165/166 and STTM165/166 were annealed in 2X SSC ( $0.3 \mathrm{M} \mathrm{NaCl}$ and $0.03 \mathrm{M}$ sodium citrate) buffer by heating at $95^{\circ} \mathrm{C}$ for $5 \mathrm{~min}$ and cooling down the mixture at room temperature for 2.5 hours. The hybridized STTM165/166-48nt-miR165/166 complexes were resolved on a $15 \%$ denatured PAGE gel for the sequestering assay. The gel was dried in a gel drier with the help of a blot and Saran, and then exposed to a phosphor-image plate, the results was 
scanned with a Typhoon 9410 Scanner and quantified by ImageQuant TL1 Software.

\section{Small RNA sequencing}

Total RNA was isolated from the leaves of 3-week-old seedlings. The small RNA was fractionated using 15\% PAGE gel electrophoresis. The small RNAs of 15-30 nt were purified from the gel and ligated to a 3' Solexa DNA adaptor. The 3' DNA adaptor ligated product was purified following the instructions. The purified 3' Solexa ligated small RNA was then ligated to a 5' Solexa RNA adaptor. The final small RNA libraries were obtained by reverse transcription. Small RNA sequencing results were conducted using Genome Analyzer II (Illumina) as previously described [53].

\section{Statistical analysis}

All results were presented as mean \pm Standard. Statistical significance was determined by unpaired Student's t-test 
(two-tailed) and ANOVA analysis. Gene expression was assayed at least for three independent experiments.

\subsection{Results}

\subsubsection{Blocking miR165/166 activity by STTM transcript in vivo}

Based on the fact that Target Mimicry (TM) functionally blocks miRNA through sequestering the miRNA from its targets and some miRNAs such as miR165/166 were not effective in using TM technology, we tried to improve the TM technology by designing an artificial transcript that could block endogenous small RNA more efficiently. By modifying the single miRNA binding site of the TM to a tandem structure with a unique linker between the two miRNA binding sites, we produced a 108nt short non-coding RNA (sncRNA) and named it short tandem target mimic (STTM). We chose a well-defined miR165/166 family, which targets and regulates the 
homeodomain/Leu zipper (HD-ZIP III) transcription factors including PHABULOSA (PHB), PHAVOLUTA (PHV), REVOLUTA (REV), ATHB8 and ATHB15 [16, 54, 55]. The transgenic plants showed dramatic phenotypic changes in leaf asymmetry, stem, flower, and vascular structure when the expression of miR165/166 was knocked down by the STTM. These phenotypes were much like the Arabidopsis phb-ld mutant line, a gain of function mutant in $\mathrm{PHB}$, on which the miR165/166 binding site was in frame mutated by a short insertion, making it uncleaveable by the miR165/166[56].

The STTM165/166 transcripts contain two miRNA binding sites, up-stream for miR165 and down-steam for miR166, linked by a 48-nt spacer. A 3-nucleotide CTA bulge in the central region of the miRNA binding site was adapted from TM technology to prevent cleavage of STTM by the miR165/166 (Fig. 1.1A). The 2X35S constitutive promoter from Cauliflower mosaic virus was used to over-express the STTM165/166 transcripts for more effective blockage of the expression of miR165/166. 
Compared with control plant, the STTM165/166-48 transgenic plant exhibited pleiotropic developmental defects comparable to the phb-ld mutant plant (Fig. 1.1B). About 90 percent of the transgenic plants exhibited strong phenotype in transgenic plants of the T1 generation: loss of apical dominance and leaf asymmetry, defection of flower organ, while the control line, expressing the empty vector without STTM structure, showed no obvious phenotype. These observations indicate that the phenotypes associated with transgenic plant were caused by STTM165/166-48. These plant phenotypes were also inheritable from generation to generation. Taken together, our results suggest the STTM can effectively block miR165/166 activity in Arabidopsis.

To determine whether these phenotypes were induced by the blockage of miR165/166, we checked the expression of miR165/166 and its five target genes (PHB, PHV, REV, ATHB8, ATHB15) at the post-transcriptional level using qRT-PCR. While miR165/166 was drastically down regulated, 
the miR165/166 target genes were up regulated in the STTM transgenic plants, but not the empty vector control transgenic plants (Fig. 1.1D), the primers used for the qPCR is listed in Table 2. Compared with the TM technology generated transgenic plants of target mimic of miR165 (MIM165), the STTM165/166 showed a much stronger phenotypic change. (Fig.1.1B).
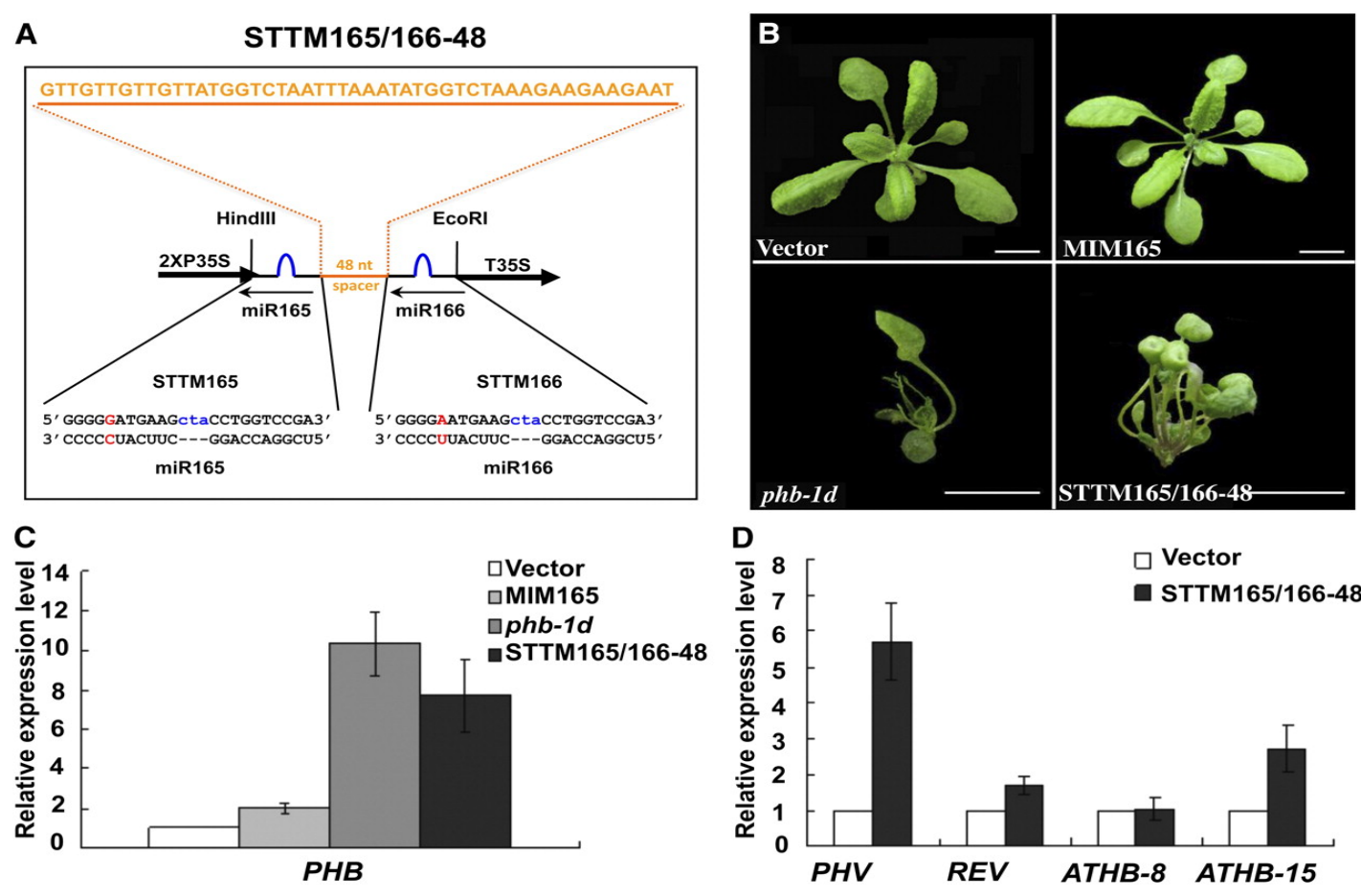

Figure 1.1. STTM165/166-48 Induced Dramatic Alterations in Arabidopsis Development. (A) Diagram of STTM165/166-48 structure showing the design strategy. Orange indicates the spacer region and the spacer sequence. Blue indicates the bulge sequences in the miRNA binding sites. Red indicates the nucleotides that are different between miR165 and miR166. nt, nucleotides. (B) Phenotypes of 
3-week-old STTM165/166-48 transformants compared with vector control (Columbia-0), MIM165, and phb-1d plants. phb-1d is a dominant genetic mutant of the PHB (AT2G34710) gene in the Landsberg erecta background, and this mutation abolishes the binding of miR165/166 to PHB mRNA. Bars $=1.0 \mathrm{~cm}$. (C) qRT-PCR analysis of the target gene PHB in STTM165/166-48 transformants compared with vector control, MIM165, and phb-1d plants. Bars show SE. (D) qRT-PCR analysis of selected targets of miR165/166 in STTM165/166-48 transformants. Bars show SE.

Table 2. Real-time PCR primers for mRNA and miRNA used in this study

\begin{tabular}{|c|c|c|}
\hline Gene Name & Real-time PCR Primer Name & sequences \\
\hline \multirow[b]{2}{*}{ PHB } & PHB-real-new-PF & \\
\hline & PHB-real-new-PR & \\
\hline \multirow[b]{2}{*}{ PHV } & PHV-real-new-PF & \\
\hline & PHV-real-new-PR & \\
\hline \multirow[b]{2}{*}{ REV } & REV-real-new-PF & \\
\hline & REV-real-new-PR & \\
\hline \multirow[b]{2}{*}{ ATHB8 } & AT4G32880-ATHB-8-real-PF & \\
\hline & AT4G32880-ATHB-8-real-PR & \\
\hline \multirow[b]{2}{*}{ ATHB15 } & AT1G52150-ATHB-15-real-PF & \\
\hline & AT1G52150-ATHB-15-real-PR & \\
\hline \multirow[b]{2}{*}{ STTM } & STTM-common-real-PF & \\
\hline & STTM-common-real-PR & \\
\hline \multirow[b]{2}{*}{ ACTIN2 } & ACTIN2-real-PF & \\
\hline & ACTIN2-real-PR & \\
\hline \multirow[b]{2}{*}{ Pri-MIR319a } & Pri-MIR319a & GGAATATATATGTAGAGAGAG \\
\hline & & GGAATACAAAAGAGAGAGGGA \\
\hline \multirow[b]{2}{*}{ Pri-MIR319b } & Pri-MIR319b & TAGATGTTGAGTTGGTGGAGG \\
\hline & & GTGGTACTCTGAGAAAGATAA \\
\hline \multirow[b]{2}{*}{ Pri-MIR319c } & Pri-MIR319c & AGCAATAAGTGCATGTGTTTGAAAC \\
\hline & & GAGTCGGCAGATATTTCTACCCTC \\
\hline \multirow{3}{*}{ Mature-MiR319 } & miR319-RT & CTCAACTGGTGTCGTGGAGTCGGCAATTCAGTTGAGTTAGCTCC \\
\hline & $\operatorname{miR} 319-\mathrm{F}$ & ACACTCCAGCTGGGACTGGATGACGCGGG \\
\hline & Stem-loop common Rev & TGGTGTCGTGGAGTCG \\
\hline \multirow[b]{2}{*}{ Mature-miR166 } & miR166-Stem-loop-RT & CTCAACTGGTGTCGTGGAGTCGGCAATTCAGTTGAGggggaatg \\
\hline & miR166-Stem-loop-F & ACACTCCAGCTGGGtcggaccaggcttca \\
\hline
\end{tabular}




\subsubsection{The spacer length between two miRNA binding sites is important}

To test if the length of the spacer in STTM transcript can affect the efficiency of the STTM, we generated more STTM165/166 with variations in the spacer length from 8 to $48 \mathrm{nt}(8,31,48$ nucleotides) (Fig. 1.2A). The transgenic STTM plants with different spacers were screened and phenotypically scored for the effect of the spacer length in inducing the phenotypic alternation.

We examined the morphology of the transgenic plants in three stages. The phenotypes of STTM165/166-48nt were compared with those of the vector control, STTM165/166-8nt, and STTM165/166-31nt (Fig. 1.2B). After germination, the STTM165/166-48nt exhibited stronger phenotypic alterations such as spoon-shaped cotyledons and alteration in the polarity of the true leaves as compared with those of the 
STTM165/166-31nt. . Interestingly, the STTM165/166-8nt with the shortest spacer had no phenotypic changes at all, resembling the control plant transformed with the empty vector (Fig. 1.2B).

Our results suggest that STTM with longer spacer exhibited better efficacy than the shorter one. We further designed longer spacer STTM with 88 and 96 nucleotides (Fig. 1.3A). Compared with STTM165/166-48nt, the STTM165/166-88nt transgenic plants exhibited severe phenotype, the transgenic plants displayed a typical loss of leaf asymmetry and pleiotropic developmental defects, but extending the spacer length to 96 nucleotides did not improve the efficiency by phenotypic scoring (Fig. 1.3B).

To investigate whether the spacer can affect STTM transcript stability that may further influence the STTM efficiency, we performed thermodynamic analysis of the structures for STTM transcripts with $8 \mathrm{nt}, 31 \mathrm{nt}, 48 \mathrm{nt}, 88 \mathrm{nt}$ and $96 \mathrm{nt}$ spacer length (Fig. 1.4). STTM $165 / 166-8$ nt RNA was apparently 
thermodynamically unstable with a $\mathrm{dG}$ of $-11.7 \mathrm{kcal} / \mathrm{mol}$, while STTM transcripts with longer spacers show higher stability $(\mathrm{dG}<-20 \mathrm{kcal} / \mathrm{mol})$. Nevertheless, thermodynamic stability may partially explain the phenotypic alteration under certain thresholds, as STTM with 31nt spacer was a little bit more stable than STTM with 48nt spacer after thermodynamic stability analysis (Fig. 1.4).

We further made more mutations on the STTM165/166-48nt in the spacer secondary structures without changing the spacer length of 48 nt (Fig. 1.5). Compared with STTM165/166-48nt that contains a weak "stem-loop" on the spacer, the STTM165/166-48nt-muts with changes in the weak "stem-loop" showed lower efficiency in inducing the production of the abnormal phenotypes. Taking into consideration the above results, we believe that both the thermodynamic stability and the spacer structure of STTM transcripts contribute to STTM efficacy. 
A

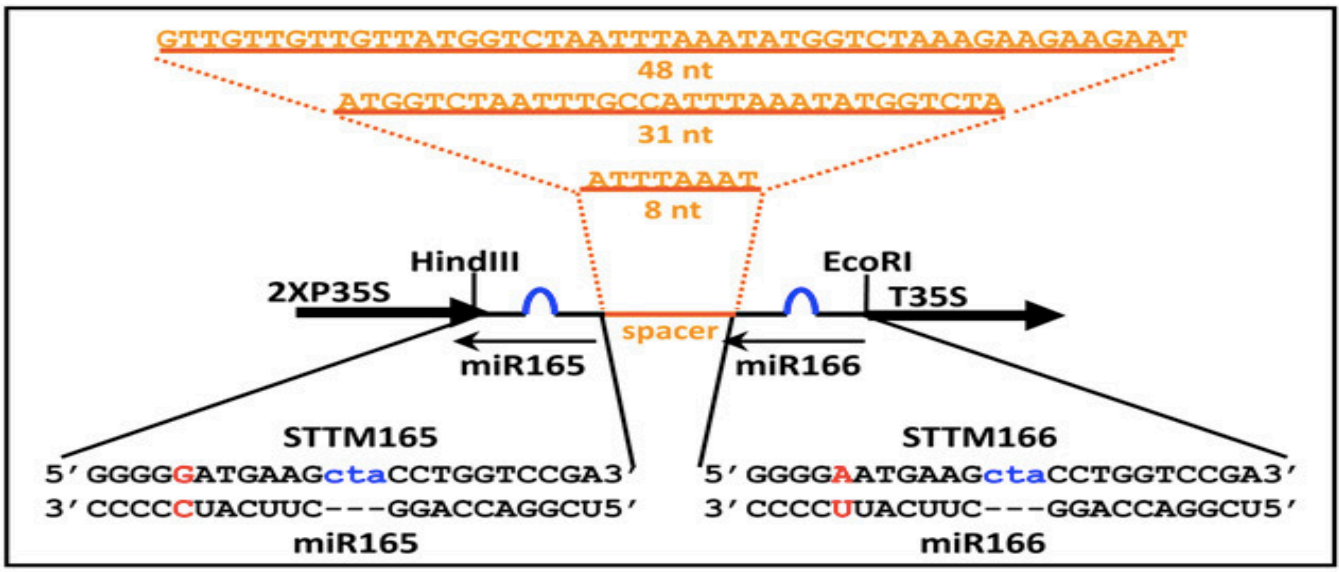

B

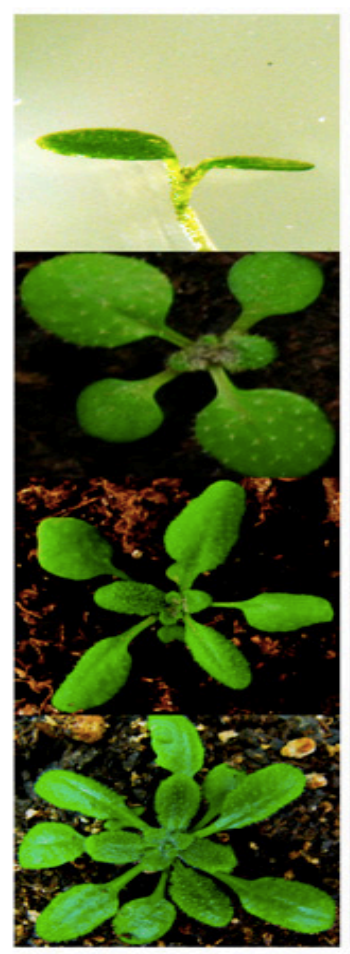

vector

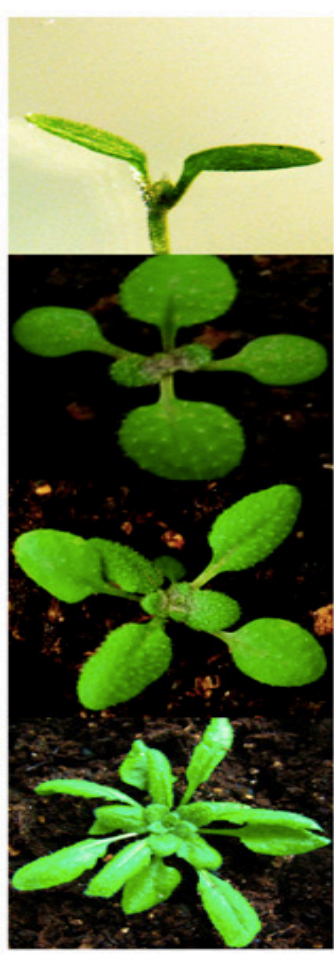

8 nt

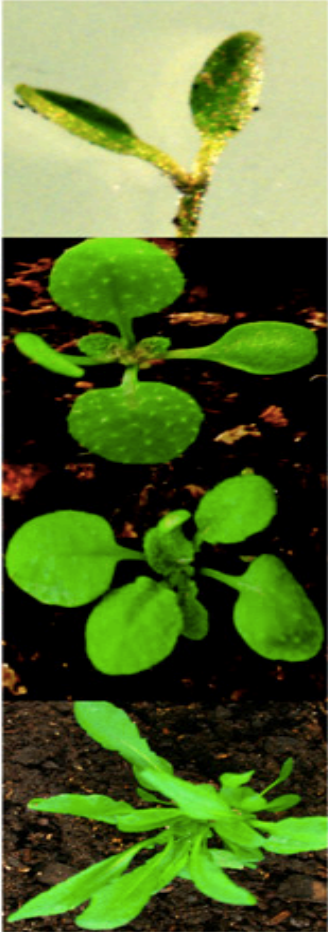

$31 \mathrm{nt}$

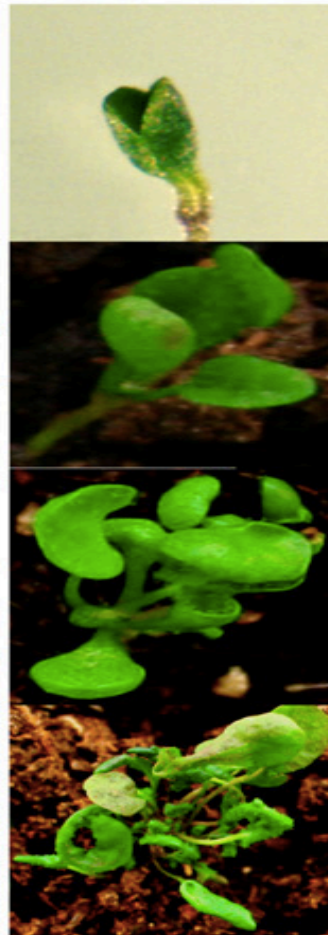

$48 \mathrm{nt}$

STTM165/166 spacer length

Figure 1.2. The Length of the RNA Spacer between the miR165 and miR166 Complementary Regions Is Crucial for STTM165/166 Function. (A) Diagrams of the STTM structures with varying lengths of the spacer region. Orange indicates the spacer region and the spacer sequence. Blue indicates the bulge sequences in the miRNA binding sites. Red indicates the nucleotides that are different between miR165 and miR166. (B) The phenotypes of representative STTM165/166 
transgenic plants with different lengths of the RNA spacers at different developmental stages. Rows 1 to 4 represent plants at days 4, 8, 15, and 30, respectively. nt, nucleotides.

A.

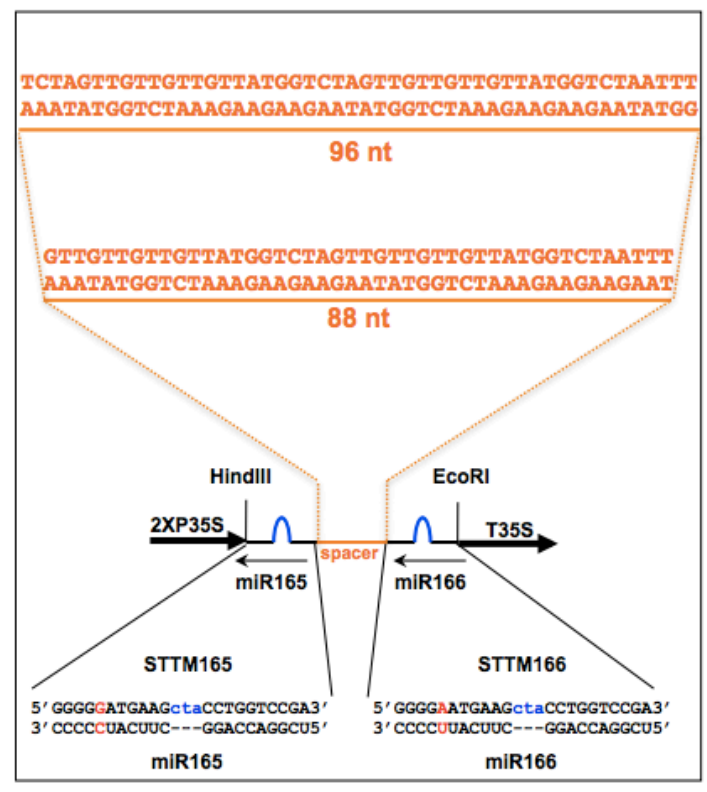

B.

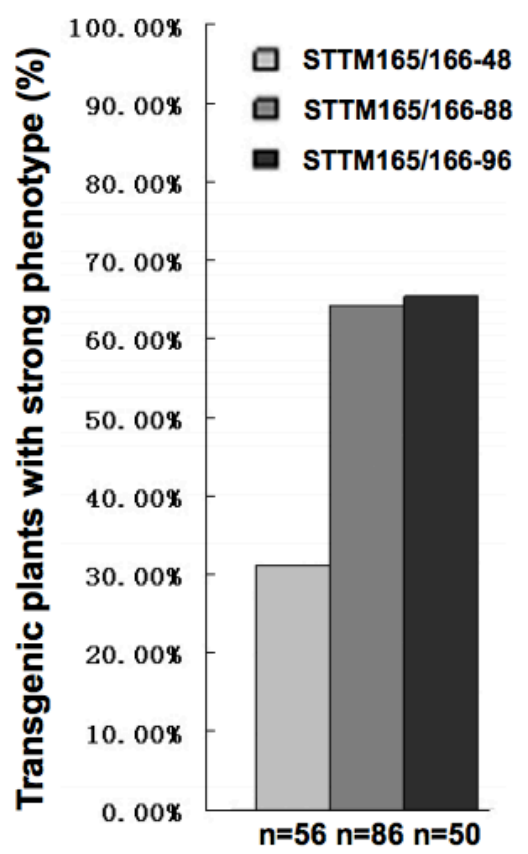

Figure 1.3. The optimal length of the spacer between the two small RNA binding sites in STTM. (A) Diagrams of the STTMs with different spacer lengths. Orange indicates the spacer region and the spacer sequence. Blue indicates the "bulge" sequences in the miRNA binding sites. Red indicates the nucleotides that are different between miR165 and miR166. (B) Comparison of the efficacy of the STTMs with different spacer lengths. 


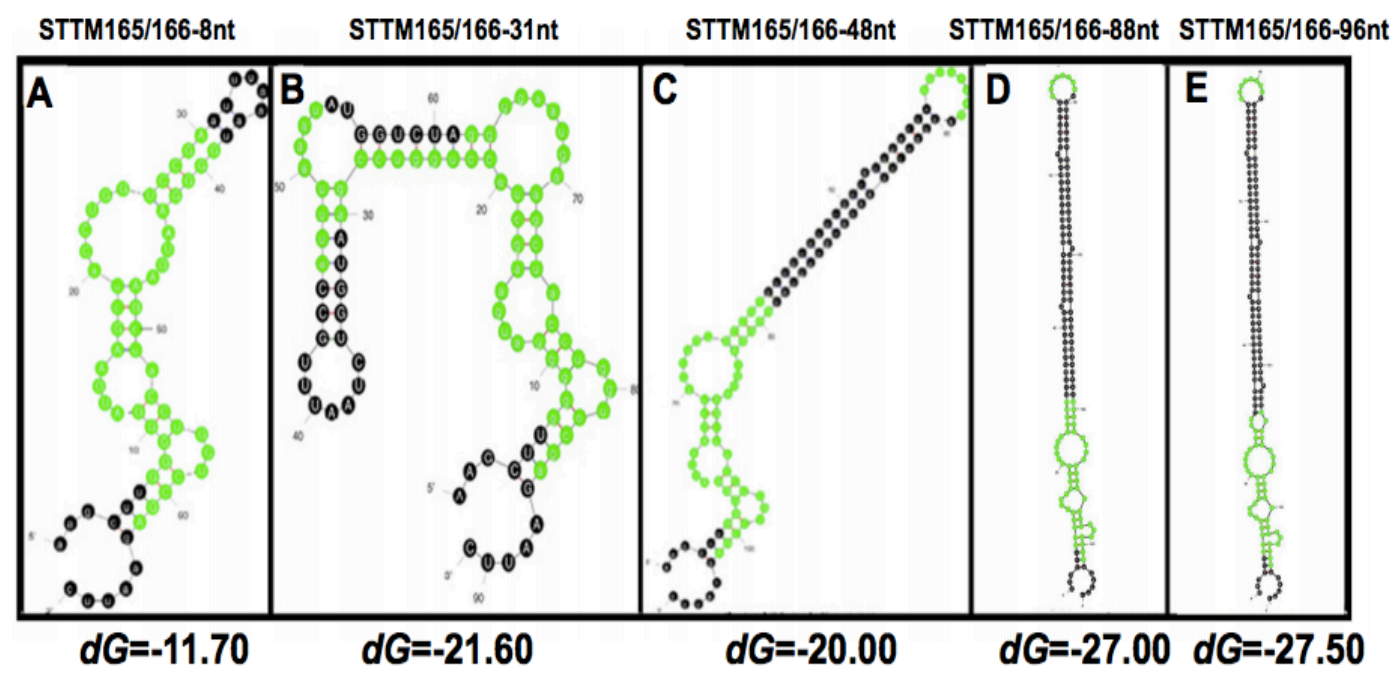

Figure 1.4. The secondary structures and thermodynamic stabilities of various STTM165/166s with different spacer lengths (8nt, 31nt, 48nt, 88nt, and 96nt). Green indicates miR165/166 binding sites or the shared portion (AUUUAAAU) of the spacer region. Black indicates the spacers or the six nucleotides of the 5' and 3' ends adjacent to the miRNA binding sites. $\mathrm{dG}$ indicates the thermodynamic stability of the STTM165/166 and the unit is $\mathrm{kcal} / \mathrm{mol}$. The lower the $\mathrm{dG}$, the more stable the STTM165/166. STTM165/166-31 and STTM165/166-48 are more stable than STTM165/166-8. STTM165/166-88 and STTM165/166-96 are more stable than STTM165/166-31 and STTM165/166-48. The spacers of STTM165/166-48 (C),-88 (D), and -96 (E) form a stable "stem" region, while those of STTM165/166-31 (B) and STTM165/166-8 (C) do not. 


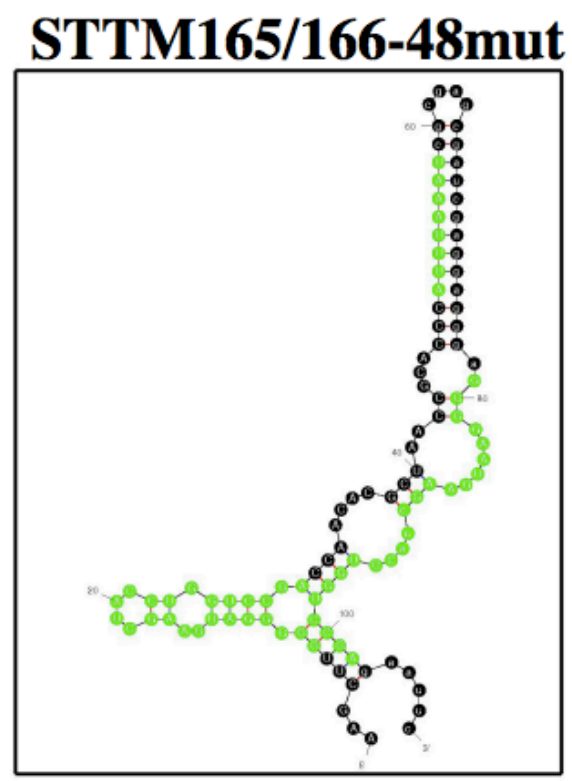

\section{STTM165/166-48mut spacer sequence: CCAACACGCUAACCGCACCC UUUAAAU CGCGAGCGAUCGAGGAGGGA}

Figure 1.5. Mutation in the spacer region changed the "Stem" structure and reduced the efficacy in the functional blockage of miR165/166. The spacer of STTM165/166-48 was randomly mutated except the AUUUAAAU region, resulting in STTM165/166-48mut. Green indicates miR165/166 binding sites or the shared portion (AUUUAAAU) of the spacer region. Black indicates the spacer or the six nucleotides of the 5' and $3^{\prime}$ ends adjacent to the miRNA binding sites. Compared with STTM165/166-48, the "stem" structure was disrupted in STTM165/166-48mut and the proportion of STTM165/166-48mut transgenic plants with strong abnormal phenotype was reduced approximately from $30 \%$ to around $20 \%$.

\subsubsection{STTM165/166-48nt transcripts induced miR165/166 degradation}


Recent studies indicate that target mimicry also triggered reduction of miRNA in addition to sequestration [57]. To investigate the mechanism that how STTM block miRNA function. We not only conducted Northern blot to detect miR165/166 level in STTM165/166 plants, but also assayed the expression level of miR165/166 in MIM165 as control. We noticed that miR165/166 reduction in MIM165 transgenic plants was only modest. In contrast, dramatic reduction in miR165/166 level was observed in STTM165/166-31nt and STTM165/166-48nt transgenic plants. There was no difference of the miR165/166 level in STTM165/166-8nt transgenic plants compared with the vector control plants (Fig. 1.6), the sequence of the probes are listed in Table 3. The expression levels of miR165/166 correlated with the severity of the phenotypic alterations in transgenic plants.

To rule out the possibility that reduction of miR165/166 in STTM transgenic plants was due to potential sequestering of miRNAs by the STTM in our Northern blotting system (Fig. 1.7B), we conducted in vitro miR165/166 sequestering 
experiment by synthetic STTM transcript and the miR165/166. In this sequestering assay, the STTM165/166 and miR165/166 were labeled by $\mathrm{P}^{32}$ to check the physical binding interaction between the synthetic miRNA and STTM. The results clearly indicated that STTM165/166-48nt was unable to sequester miR165/166 under conditions similar to our Northern blotting process.

To further confirm miR165/166 reduction in tissues of STTM165/166-48nt plants, we generated small RNA libraries from the total RNA of the STTM165/166-48nt transgenic plant as well as vector control plant. The miRNA reads were first collected by filtering away other types of small RNAs, and then normalized to 9 million of the total small RNA reads. MiR165, miR166, miR159 and miR168 reads were calculated and compared between the STTM165/166-48nt and the vector control plants. Our informatics data indicated that the reads of miR168 and miR159 were about at the same level between STTM165/166-48nt and vector control. Significantly, the reads of miR165 and miR166 of STTM165/166-48nt were 
dramatically reduced relative to the vector control (Fig. 1.7C). This result further confirmed the reduction of miR165/166 induced by STTM165/166 in vivo.

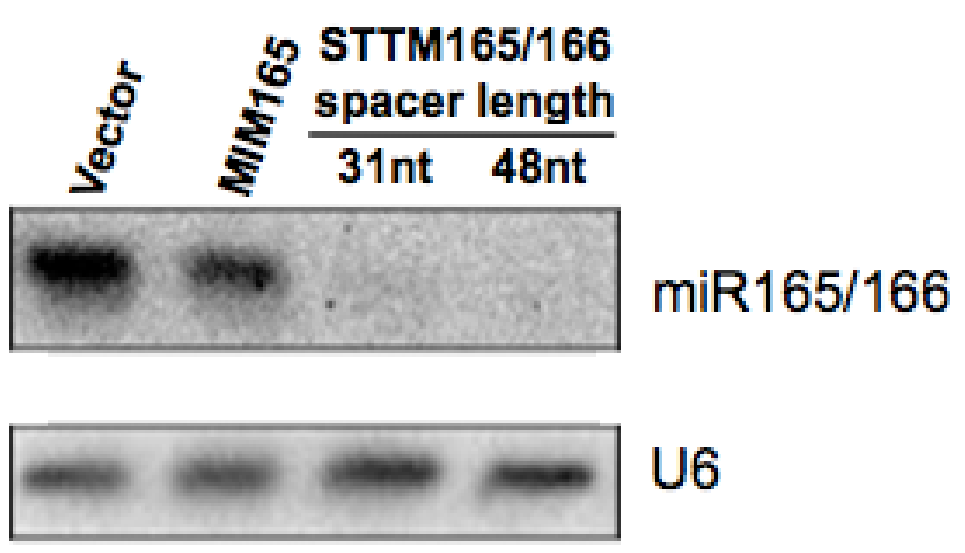

Figure 1.6. Comparison of the expression levels of miR165/166 in STTM165/166 and MIM165 transformants and vector control plants. miR165/166 and U6 was assayed by RNA gel blotting analysis. 
A
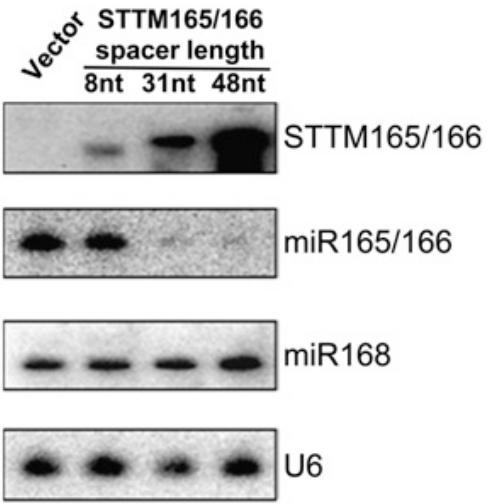

C $\quad$ miR165

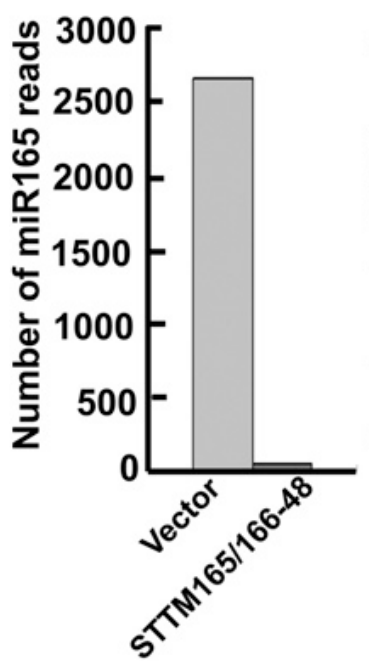

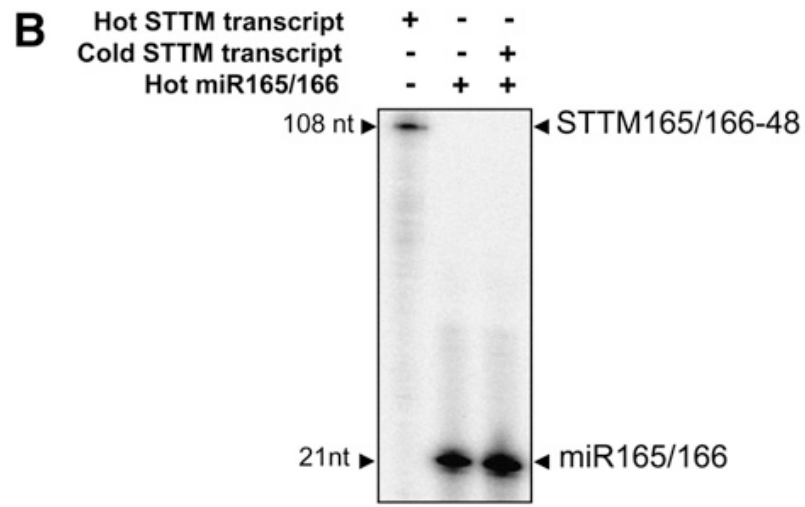

$\operatorname{miR} 166$

$\operatorname{miR} 159$

miR168
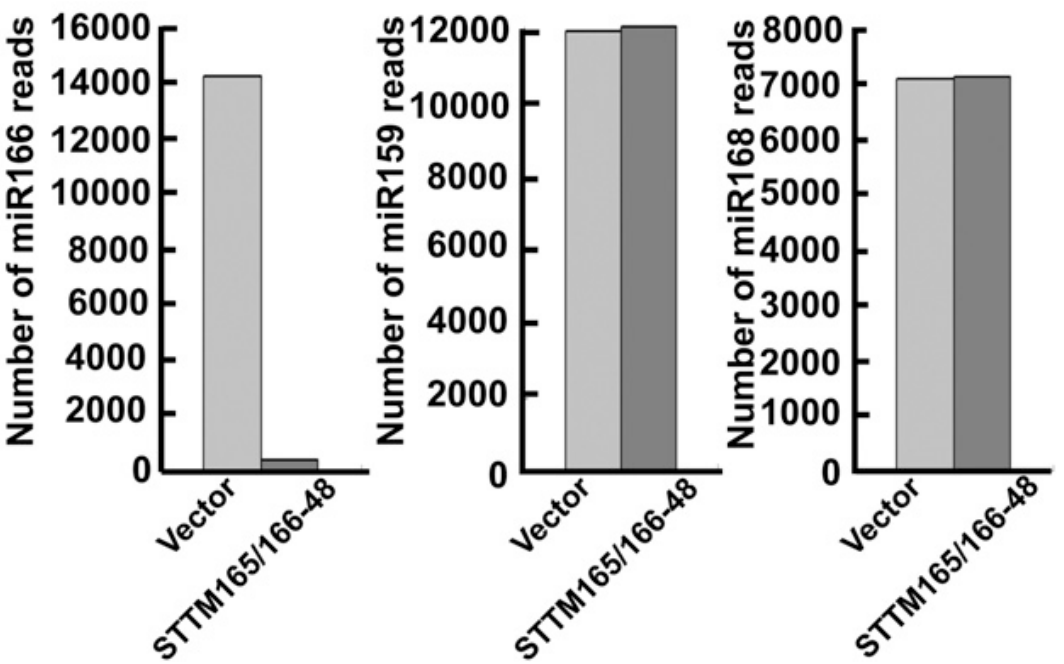

Figure 1.7. STTM165/166 Triggered Drastic Reduction of miR165/166 Levels. (A) Representative RNA gel blotting to detect STTM165/166, miR165/166, and miR168. U6 served as an internal control. nt, nucleotides. (B) STTM165/166-48 was unable to affect the migration of miR165/166 on a denaturing polyacrylamide gel. (C) Copy numbers of small RNA reads in vector control and STTM165/166-48 by deep sequencing. 
Table 3: Synthetic miRNA and probes used in this study

\begin{tabular}{|c|l|l|}
\hline \multirow{4}{*}{ Probe/RNA } & 01igo/Probe name & Sequences \\
\hline \multirow{4}{*}{ miR165/166 } & miR165-probe & GGGGGATGAAGCCTGGTCCGA \\
\cline { 2 - 3 } & miR166-probe & GGGGAATGAAGCCTGGTCCGA \\
\hline \multirow{2}{*}{ miR156/157 } & miR156-probe & GTGCTCACTCTCTTCTGTCA \\
\cline { 2 - 3 } & miR157-probe & GTGCTCTCTATCTTCTGTCAA \\
\hline \multirow{3}{*}{ Synthetic miR165/166 } & miR165-RNA & UCGGACCAGGCUUCAUCCCCC \\
\hline \multirow{2}{*}{ miR168 } & miR168-probe & TTCCOGACCTGCACCAAGCGA \\
\hline \multirow{2}{*}{ miR319 } & miR319-probe & GGGAGCTCCCTTCAGTCCAA \\
\hline U6 & U6-probe & GCTAATCTTCTCTGTATCGTTCC \\
\hline
\end{tabular}

\subsubsection{Blocking other miRNAs in Arabidopsis by STTM}

To investigate whether STTMs can serve a general tool to be applied to block miRNA in plants, we designed additional primers to construct STTMs to block miR156/157, and miR319 in Arabidopsis (Fig. 1.8). The sequence of miR156 and miR157 are different by 3 nucleotides (Fig. 1.8A), and they share the same targets-SQUAMOSA PROMOTER BINDING PROTEIN-LIKE (SPL) transcription factors, 
including SPL3, SPL4, SPL5. Based on previous studies, SPL family genes can promote vegetative phase transitions[58], which can serve as a good indicator to assay STTM efficiency in functional blocking of miR156/157.

STTM156/157-48nt transgenic plants exhibited delayed leaf initiation in early stage and advanced flower timing (Fig. 1.8B). STTM156/157 plants bolted with only about six leaves, while the control plants need longer time to flower at about the 10-leave stage (Fig. 1.8B). The small RNA gel blotting result indicated that miR156/157 was decreased significantly in STTM156/157-48nt transgenic plants, as compared with vector control plants (Fig. 1.8C).

We similarly designed STTM319-48nt to block miR319 expression in Arabidopsis (Fig. 1.9). MiR319 is involved in plant leaf development, affecting leaf morphogenesis and leaf senescence [59]. MiR319 regulates the transcription factors of TCP family as its targets. High levels of miR319 or low TCP activity causes accelerated cell proliferation[60]. In contrast, 
the STTM319-48nt transgenic plants appeared shorter petiole and dark green leaf compared with control transgenic plants (Fig. 1.9A). To further determine the miR319 expression in STTM319-48nt plants, we conducted RNA gel blotting analysis of the miR319 expression, and the result showed that the miR319 was reduced by about $95 \%$. The miR319 targets of the TCP family were determined by qRT-PCR and the data showed that targets TCP2, TCP3, TCP4, TCP10, TCP24 were significantly up regulated in the STTM transgenic plants as compared to the control plant (Fig. 1.9B, Fig. 1.9C).

Taking all the above results together, we concluded that STTM can serve a general tool for functional analysis of miRNAs in Arabidopsis and that blocking miRNAs by STTM induces the up regulation of the miRNA target genes. 


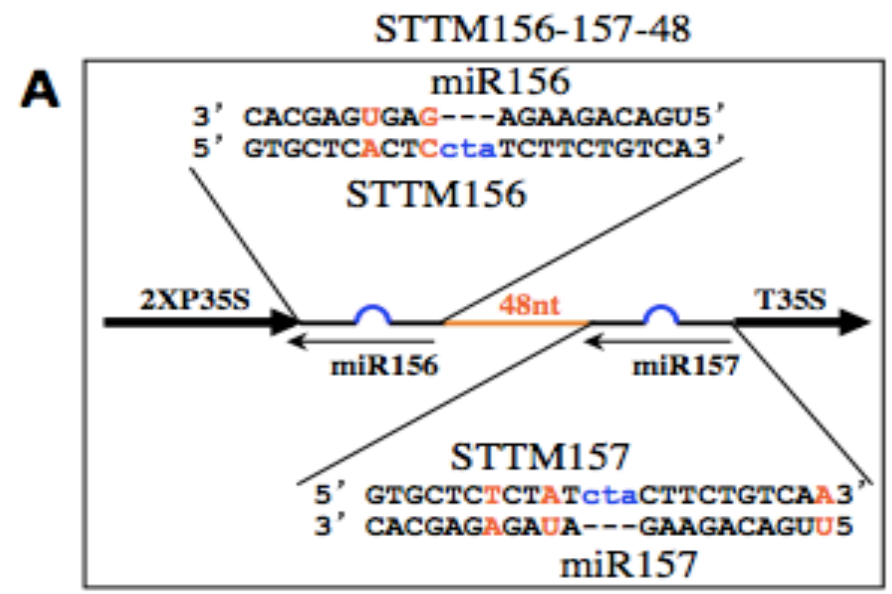

$\mathbf{B}$

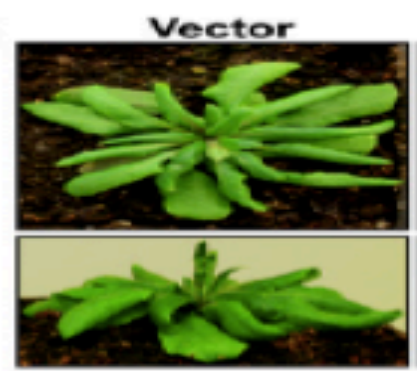

STTM156/157-48
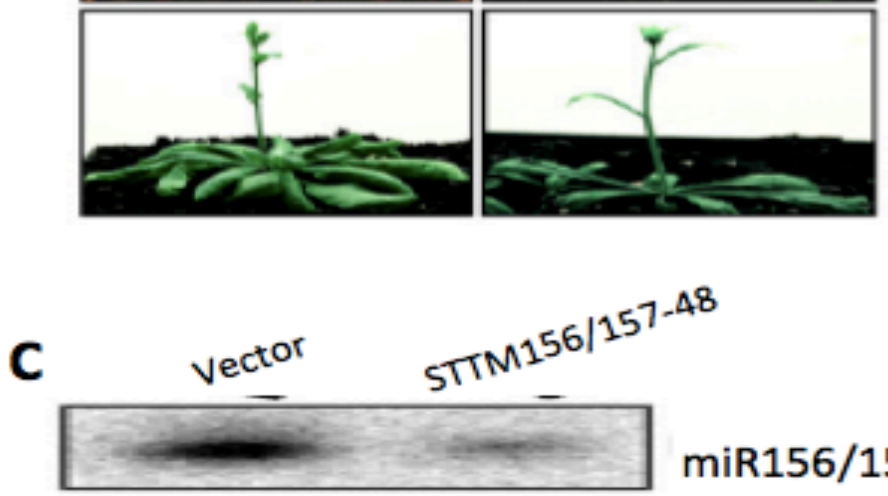

$\operatorname{miR} 156 / 157$

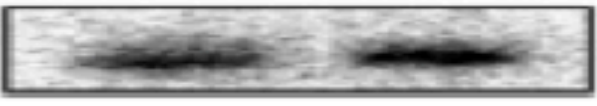

U6

Figure 1.8. STTM is effective in triggering the reduction of miR156/157. (A) Diagram of STTM156/157 and the miR156/157 binding sites. (B) Phenotypes of STTM156/157 transgenic plants. (C) Northern blot detection of the miR156/157 expression level in vector control and STTM156/157-48nt transgenic plants; U6 was used as a loading control. 


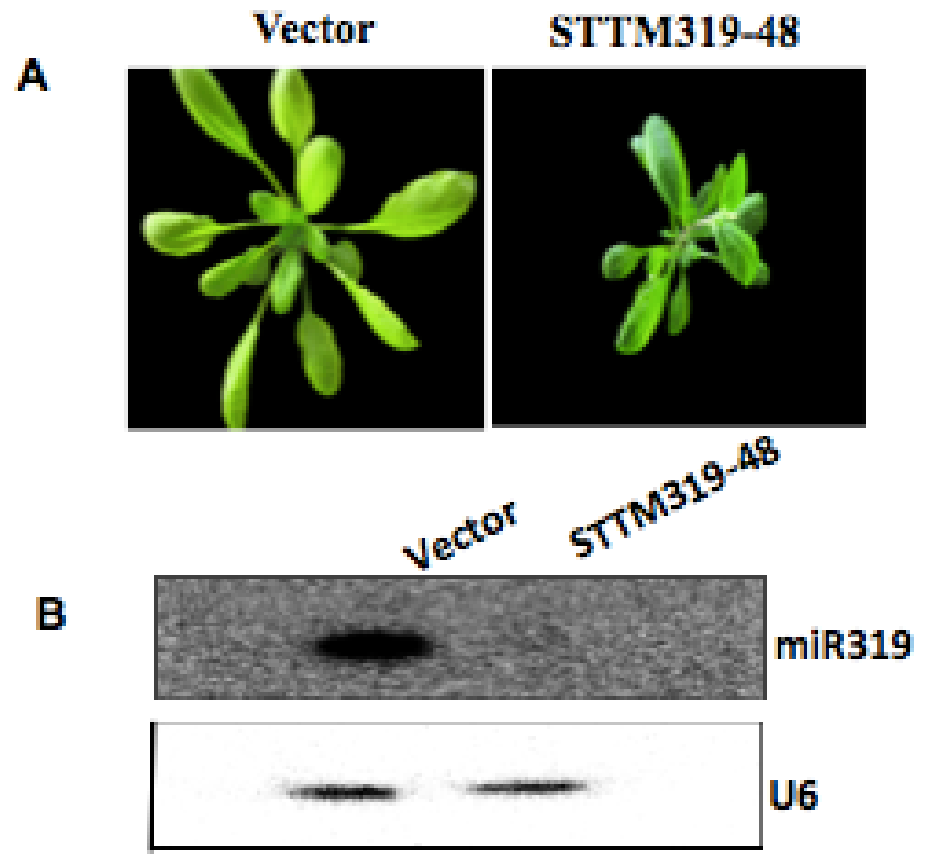

Figure 1.9. STTM is effective in triggering the reduction of miR319. (A) Phenotypes of STTM319 transgenic plants (right) and the vector control (left). (B) Small RNA gel blotting result of the vector control and STTM319-48nt transgenic plants. Row 1 is for miR319 signal and row2 as the U6 loading control.

\subsubsection{SDNs play an important role in STTM induced miRNA degradation}

Previous research indicated that the SDNs exonucleases were turnovering small RNAs in vivo [61]. To test if SDNs contribute to the miRNA degradation triggered by STTM 
transcripts, we transformed STTM165/166-48nt into $s d n 1 / s d n 2$ plant (Fig. 1.10A). The $s d n 1 / s d n 2$ plants appeared no obvious phenotypes compared with the wild-type control plants [61]. After transforming with STTM165/166-48nt, most transgenic plants in the background of the $s d n 1 / s d n 2$ double mutant showed no phenotype compared to the vector control plants. Only a small fraction of the $\mathrm{T} 1$ transgenic plants with STTM165/166 in $s d n 1 / s d n 2$ background showed modest phenotype. In contrast, about 90 percent of STTM165/166-48nt in wild-type background exhibited severe phenotypes. We further determined the STTM165/166-48nt transcripts and miR165/166 expression pattern of STTM165/166-48nt transgenic plants both in wild type and $s d n 1 / s d n 2$ backgrounds (Fig. 1.10B). The RNA gel blotting results indicated that the STTM165/166-48nt transcripts were similar in the $s d n 1 / s d n 2$ and wild type backgrounds, but the miR165/166 expression level in $s d n 1 / s d n 2$ background was significantly higher compared with the transgenic plants in wild type background (Fig. 1.10B). We further identified two individual STTM165/166-48nt transgenic plants in the 
$s d n 1 / s d n 2$ background. We detected the STTM165/166-48nt transcripts and miR165/166 levels in two independent lines containing the STTM165/166-48nt in $s d n 1 / s d n 2$ background, and the results showed that the STTM transcripts levels were comparable in the two independent STTM165/166-48nt transgenic lines with $s d n 1 / s d n 2$ background, as compared with the wild type background (Fig. 1.10C, Fig. 1.10D). Although the STTM transcripts were comparable, the miR165/166 expression level was significantly high in the transgenic plant of the $s d n 1 / s d n 2$ background, as compared to the transgenic plants of wild type background plants. In summary, the SDN gene family contributes to the miRNA degradation induced by STTM. 


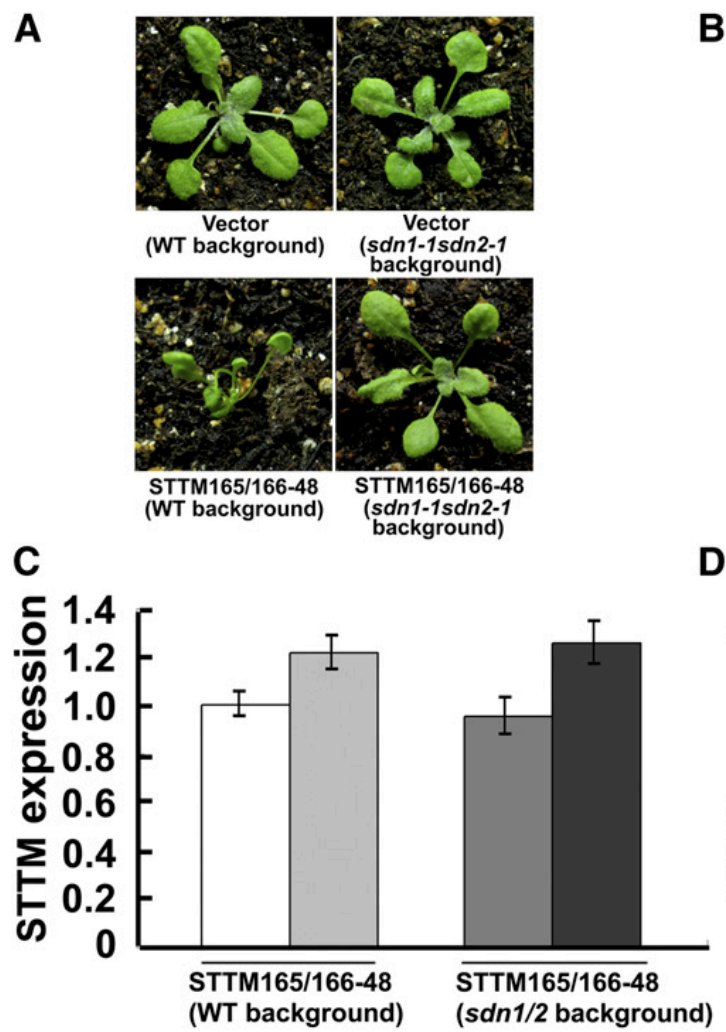

B

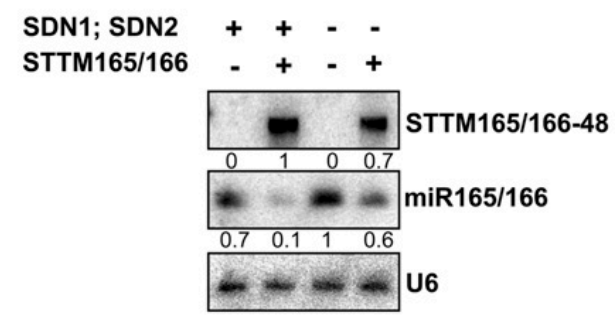

D

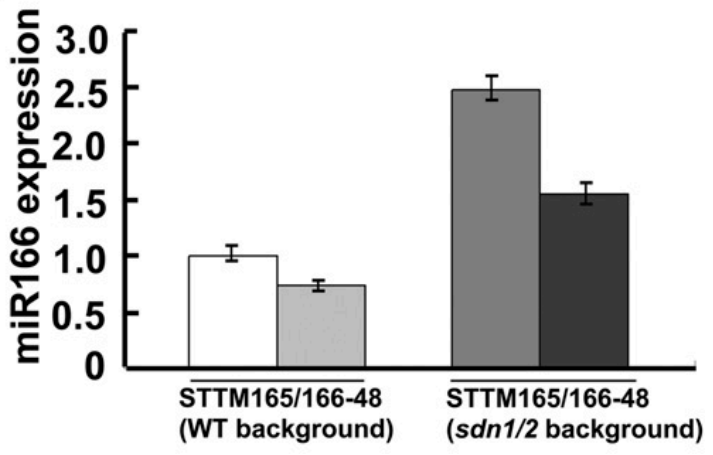

Figure 1.10. Degradation of miR165/166 by the SDNs contributes to the reduction of miR165/166 triggered by STTM in Arabidopsis. (A) Phenotypes of vector and STTM165/166-48 transgenic plants in the sdn1-1 sdn2-1 background (at right) compared with those in the wild type (WT) background (at left). (B) RNA gel blotting to determine the levels of STTM165/166-48 RNA, miR165/166, and the U6 control RNA in the wild type (SDN1; SDN2 +) or sdn1-1 sdn2-1 (SDN1; SDN2 _) background. The numbers indicate the relative levels of the RNAs. (C) Comparison of STTM expression in STTM165/166-48 transgenic plants in sdn1-1 sdn2-1 background and in wild-type background. Bars show SE. (D) Comparison of miR166 levels in STTM165/166-48 transgenic plants in sdn1-1 sdn2-1 background and in wild-type background. Bars show SE. (C) and (D) Different shades of gray are used to indicate different independent lines to allow for the easy comparison between STTMandmiR165/166 levels in the same lines. 


\subsection{Discussion}

In this study, we developed a new technology termed STTM to functionally block endogenous miRNAs in Arabidopsis. The results showed that STTM can knockdown the expression of miRNAs effectively. The dramatic reduction of miRNA by STTM, in turn, induces the up regulation of the miRNA targets. Due to characteristics of miRNA target genes largely encode transcription factors as master regulators, regulation of miRNAs by STTM directly leads to the alteration of these master regulators, resulting in growth and developmental alteration in plants. Compared with previous technologies, such as miRNA overexpression, production of miRNA-resistant targets, or even the recent TM technology, in study of miRNAs, STTM has obvious advantages in many applications. First, STTM is simple in its construction by PCR, making application of large-scale STTM in different plants possible. Second, STTM contains two miRNA binding sites that are more effective than TM since TM just has one miRNA 
binding site. The linker between the two STTM modules potentially has a synergistic role in promoting miRNA degradation the STTM binds, which make it possible to target two different miRNAs for degradation simultaneously.

Recent studies have extended STTM technology to block the miRNA in other plant species, such as tomato and rice[12]. Moreover, the STTM technology was also applied in animal cells such as the MIN6 cell line to block animal endogenous miRNA successfully [62], which indicates that the STTM technology can be widely used in both plant and animal systems.

Due to its small size, just about 100 nucleotides, STTM has the potential to be arranged in multiple modules to silence multiple endogenous miRNAs in plants, and further more, STTM can be combined with other genomic tools such as gene editing, artificial miRNA (amiRNA) technologies to modulate the miRNA network in plants and animals. 
Large-scale production of transgenic STTMs in various plant species is valuable in functional study of miRNAs in plants. Such kind of resource is valuable for community sharing. Many STTM constructs are now available at Addgene.com through this and related studies, supported by National Science Foundation. Thus, the development of STTM technology contributes significantly to functional genomics of miRNAs in plants. The fact that STTM can be stably expressed in plants from generation to generation makes STTM transgenic plants more valuable in fundamental as well as applied studies. Various STTM lines in crops may serve as breeding material for crop improvement. The rather stable STTM transcripts in plants may bypass the non-sense mediated RNA decay (NMD) pathway due to its small size stabilized by a Cap at the 5 ' end and poly(A) tails at the 3' end[63]. Indeed, various endogenous non-coding RNAs have been discovered in plants in large quantities. The existence of these genetically stable non-coding RNAs suggests their importance and can possibly be manipulated as STTM to regulate gene expressions. In addition, the stability of STTMs may partially depend on their 
thermodynamic stability, enhanced by the linker structures. Such secondary structures with high stability may shed lights on the secondary structure of many non-coding RNAs in various organisms.

Most likely, STTM can trigger both the degradation of miRNAs and the sequestering of miRNAs in vivo. Such speculation is based on the observations that not all the STTMs are effective in triggering the miRNA degradation. Nevertheless, in some cases, STTM transgenic plants have phenotypes without detections of apparent miRNA degradation. We generated large-scale study that using STTM tool to trigger the degradation of other endogenous miRNAs, which is possibly mediated by SDNs. It is extremely interesting to investigate how STTM recruits SDNs to the miRNA-protein complexes and further to degrade the miRNAs. 


\section{MiRNAs can regulate plant anthocyanin accumulation in Arabidopsis}

Yiyou Gu, Mengmeng Qiao, Jun Yan, Guiliang Tang

The following chapter is part of the manuscript under preparation. 


\subsection{Introduction}

Anthocyanins belong to a parent class of molecules called flavonoids, which are synthesized via the phenylpropanoid pathway. They are water-soluble that may appear red, purple, or blue depending on the $\mathrm{pH}$ [64]. The anthocyanin is odorless, flavorful, contributing to taste as a moderately astringent sensation [64, 65]. Raspberry, black rice, soybean and blueberry are anthocyanin enriched food plants, and flowers, seeds and fruits are common diet sources of anthocyanin [66, 67]. The anthocyanins not just supply the food flavor, they also have functions in attracting pollinators, and most importantly, they are involved in process of plant growth and development $[68,69]$. In addition, many simple flavonoids have antioxidant properties, which makes the anthocyanin rich plants highly valuable for the human health $[68,70]$. Therefore, improving the anthocyanin accumulation in food plants is very important in research. The most common flavonoids in Arabidopsis are kaempferol glycosides, the level of which increases upon 
exposure to UV radiation[71]. The glycosylated and acylated cyanidin derivatives are the major anthocyanins in the Arabidopsis leaf and stem region[72].

The biochemistry and genetics of anthocyanin have been well studied since 1800 s by Mendel [73] and the enzymes that involved in anthocyanin biosynthetic pathway are well characterized [74]. Also, the anthocyanin pathway genes from different species share high similarity sequence [75].

Seven enzymes are involved in anthocyanin biosynthesis, including phenylalanine ammonialyase (PAL), chalcone synthase (CHS), chalcone isomerase (CHI), flavanone 3-hydroxylase $(\mathrm{F} 3 \mathrm{H})$, dihydroflavonol reductase (DFR), anthocyanidin synthase (ANS), and flavonol 3-glucosyltransferase (3GT) [76, 77]. CHS involved in the first step of the biosynthetic pathway. Till now, twelve CHS genes (ChsA, B, C, D, E, F, G, H, I, J, K and L) have been identified in P. xhybrida [78]. Then the chalcone is isomerized to naringenin by $\mathrm{CHI}$. Naringenin is converted to 
dihydrokaempferol by F3H. The dihydrokoempferol will be further catalyzed into dihydroquercetin by $\mathrm{F} 3 \mathrm{H}$. Part of the dihydrokoepferol and dihydroquercetin will be catalyzed by FLS to pigment flavonols and glycosidic derivatives, the remaining dihydrokeepferol and dihydroquercetin are catalyzed to by DFR as a major step of anthocyanin biosynthesis[79, 80].

The genes encoding the above-mentioned enzymes are regulated by R2R3 MYB, basic helix-loop-helix (bHLH) and WD repeat (WDR) transcription factors [81]. These three transcription factors working together, forming a trimeric MYB-bHLH-WDR (MBW) complex to directly activate DFR, ANS and 3-GT $[82,83]$. These transcription factors belong to R3 MYB and NAC families[84]. Interestingly, these transcription factors can be repressed or regulated by microRNAs (miRNAs) at the posttranscriptional level [85].

Small RNAs, including miRNAs and small interfering RNAs (siRNAs), play very important roles in growth and 
development, epigenetics, genome integrity, defense against viral infection, and response to environmental changes in plants [86, 87]. Most miRNA target genes in plants are transcriptional factors. These transcriptional factors regulate plant development and phase change at different growth and developmental stages. Several miRNAs have been reported to regulate anthocyanin biosynthesis. MiR156 target SPL9 has been indicated involved in anthocyanin biosynthesis[88]. The miR828 negatively regulates the anthocyanin accumulation through repressing the target gene expression of MYB75, MYB90, and MYB13 in Arabidopsis[85]. The miR858 can negatively regulate anthocyanin accumulation[89].

We generated several miRNA blockage lines in transgenic plants using the STTM method. As previous report, anthocyanin was repressed in STTM156/157 transgenic plants due to the up-regulated SPLs. Also, in the STTM165/166-48nt transgenic plants, the anthocyanin accumulated dramatically in the leaves compared with the vector control transgenic plants. Several miRNAs-anthocyanin regulation pathways have been 
well studied, related miRNAs including miR156/157, miR828, and miR858. How are these different miRNA-targets pairs working together to regulate the plant anthocyanin biosynthesis is an interesting topic for research. Taking advantage of the short-size STTM structure, it would be very interesting to block two or more anthocyanin related miRNAs by STTM and investigate their interactions. In this chapter, we blocked miR165/166 and miR156/157 using such strategy for better understand the roles of miRNAs in anthocyanin biosynthesis. Because of strong phenotype associated with the STTM165/166-48nt transgenic plants, we used 1X35S promoter to replace the $2 \mathrm{X} 35 \mathrm{~S}$ promoter and obtained STTM165/166 transgenic plants with milder phenotype for better analysis of the miRNAs-mediated anthocyanin biosynthesis. This newly generated STTM165/166 transgenic plants had a selection marker gene to catalyze hygromycin antibiotics. On the other hand, we blocked miR156/157 by STTM with 2 X35S stronger promoter and used the herbicide BASTA for screening transgenic plants. Transgenic STTM165/166 and STTM 156/157 were crossed and screened 
by double antibiotic selection markers to generate the hybrid with both miR165/166 and miR156/157 knocked down by STTM technology. Detailed analyses and comparisons of the parent STTMs and their hybrid are presented in this chapter.

\subsection{Experiment procedures}

\section{Construction of STTM plasmids and transgenes}

STTM165/166-48nt module was assembled between 1X35S promoter and $35 \mathrm{~S}$ terminator in $\mathrm{pOT} 2$ vector by PCR method amplification, STTM156/157-48nt module was assembled between $2 \mathrm{X} 35 \mathrm{~S}$ promoter and $35 \mathrm{~S}$ terminator in pOT2 vector by PCR amplification, as described in Chapter 3 (3.2). The STTM structures were included in the two long PCR primers ( 104 nucleotides (Table 1), SwaI restriction endonuclease site were designed on each primers. The back to back PCR product including the pOT2 backbone was purified by PCR product 
purification kit (Promega) and then digested by SwaI restriction endonuclease. After the endonuclease digestion, the products were purified by the PCR purification kit (Promega) and ligated overnight at room temperature. The ligated products were transformed using XL1-blue competent cell. Select single colonies for plasmid purification, the recombinant plasmids containing the STTM were screened and identified by SwaI endonuclease digestion. Pair of primers that contain PacI sites were further used to amplify the recombinant plasmid, and the PCR product containing the STTM structure and pOT2 backbone having the antibiotic selection marker but without the plasmid replication origin was used for cloning into the final binary vector. The modified binary vector pCAMBIA1300 containing a unique PacI site was mixed with the above PCR production containing the recombinant plasmid pOT2 backbone, 1X35S-STTM165/166-48nt, and the selection marker and then digested by PacI. The digested products were purified and ligated together with T4 DNA ligase (NEB Inc.) overnight. The ligation product was transformed into XL1-blue competent cells, and the recombinant constructs were 
identified by Chloramphenicol and Kanamycin double antibiotics selection. The well-identified recombinant plasmids through PacI cleavage were sent for DNA sequencing before applying for plant transformation.

Transgenic plants were generated by Agrobacterium tumefaciens-mediated floral dip transformation [90]. The seeds were collected after floral dip. Resistance to hygromycin on 0.5M Murashige Skoog (MS) plates was used to screen for the 1X35S-STTM165/166-48nt transgenic plants, and resistance to herbicide BASTA to screen the 2X35S-STTM156/157-48nt transgenic plants.

\section{Crossing Arabidopsis plants between the STTM165/166 and STTM156/157}

We generated the hybrid between STTM165/166-48nt and STTM156/157-48nt transgenic plants through genetic crossing.

First, we generated STTM165/166-48nt and STTM156/157-48nt transgenic plants individually and 
obtained the stable line for genetic crossing between them. Due to the strong phenotype with less fertility in STTM165/166-48nt plants, we used the STTM156/157-48nt transgenic plant as the "female" parent. We chose several young flower buds that are not open, yet not closed (right before the white petals become visible) on the STTM156/157-48nt transgenic plant for crossing with pollens from STTM165/166-48nt plants. Specifically, too old or too young flowers were removed from the inflorescence on the STTM156/157-48nt transgenic plant, leaving 2-6 flowers for pollination. We sterilize the forceps and wipe the fingers with $70 \%$ ethanol before dissecting the flower buds, then removed the sepals, petals, and stamens parts of flowers from the flower bud, without injuring the pistil and flower stalk. After done with the "female" parent, a suitable pollen-donor flower was selected from the "male" parent -STTM165/166-48nt transgenic plant. The flower that has opened and has petals perpendicular to the main flower pollen was selected for pollens to be used for crossing. The pollens from STTM165/166-48nt transgenic plant were collected and placed 
twiceonto the tip of the exposed ovary of the STTM156/157-48nt transgenic plant. The pollinated ovary was covered with saran wrap to allow its further development to maturity. The saran wrap was removed after 5 days and seeds will be harvested once the labeled crossing siliques are dried. The hybrid seeds were then grown and screening on $0.5 \mathrm{X}$ Murashige Skoog plates containing $50 \mathrm{mg} / \mathrm{mL}$ hygromycin. We growed the hybrid plants to 4 leaves stage after germination, then transferred the plants to soil, to confirm the hybrid plants with herbicide BASTA. The identified hybrid plants were used for the further analysis.

\section{Small RNA gel blotting}

Total RNAs were isolated from Arabidopsis leaves from T2 generation of transgenic and vector control plants using trizol (Invitrogen), and 10ug total RNAs were resolved in 15\% denatured PAGE gel. The $\mathrm{P}^{32}$ isotope labeled probes complementary to sequence of the miRNAs were used for Northern blotting analysis [49]. The sequences of the probes 
and miRNAs are listed in Table 3. To detect the STTM transcripts in the transgenic plants by the northern blot, the STTM constructs reverse primers were labeled by $\mathrm{P}^{32}$ isotope as probes. U6 expression was detected as internal control.

\section{Detecting mRNA expression by qRT-PCR}

After the transgenic plants were screened and confirmed by Northern blot for the degradation of miRNA, we detected the expression of the miRNA target genes by qRT-PCR. Total RNA from hybridized plants, parent transgenic plants, and vector control plants were isolated using RNeasy mini kit (Qiagen). We removed the potential genome DNA contamination by DNase before the RNA reverse transcription. Random primer was used as the reverse transcription primer. RT-PCR using the SYBR green PCR master mix kit was performed with an Applied Biosystems step one instrument $(\mathrm{ABI})$. Actin gene expression was used for the internal control. The sequences of primers used for qRT-PCR are listed in Table 2. The relative expression levels of miRNA targets were 
calculated using $2-^{\wedge}$ cycle threshold method[91]. Three biological replicates were examined for all the target detection to ensure reproducibility.

\section{In-situ hybridization and immunehisto- chemistry staining}

Leaves and stems from transgenic "male" parent, "female" parent, and the hybrid plants were dissected and fixed in $4 \%$ formaldehyde $\left(\mathrm{pH}\right.$ 7.4) overnight at $4{ }^{\circ} \mathrm{C}$ and then processed routinely for paraffin embedding. Tissues were cut into $10 \mu \mathrm{m}$ sections and adhere to glass slides (Superflost, Fisher Scientific). For in-situ hybridization, sections were first deparaffinized and rehydrated, then treated with Proteinase K (Roch, $40 \mu \mathrm{g} / \mathrm{ml}$ ) as described [92]. Briefly, a total of 3 pmol of DIG-labeled Locked Nucleic Acid (LNA) probes (Exiqon) were mixed with $200 \mu \mathrm{l}$ of hybridization buffer and applied onto the slides in order to hybridize at $37^{\circ} \mathrm{C}$ for overnight. Slides were then washed using 2X SSC (saline-sodium citrate) solution and incubated with alkaline phosphatase-conjugated 
sheep anti-DIG antibody (Roche) at $4^{\circ} \mathrm{C}$ for overnight. Alkaline phosphatase reaction was carried out with $50 \mathrm{mg} / \mathrm{ml}$ of NBT/BCIP staining solution at room temperature for 1-3 days. The images were captured on Olympus FluoView FV1000 confocal microscopy.

\section{Statistical analysis}

All results are expressed as mean $\pm \mathrm{SD}$. Statistical significance is determined by unpaired Student's t-test (two-tailed) and ANOVA analysis. Each gene expression was checked at least for three independent experiments.

\subsection{Results}

\subsubsection{Functional blockage of both miR165/166 and miR156/157 by crossing between STTM165/166-48nt and STTM156/157}


Due to strong phenotype with less seed productivity from STTM165/166-48nt transgenic plants with 2 X35S promoter, we generated transgenic plants of the STTM165/166-48nt with $1 \mathrm{X} 35$ constitutive promoter, which had a mild phenotype with higher productivity (Fig. 2.1A). Transgenic plants exhibited anthocyanin repression in both the leaf and the stem region (Fig. 2.1B). The flowering was accelerated in the STTM156/157-48nt plants just with only six leaves, while the vector control transgenic plants and wild-type plants normally bolted after ten leaves. Because the STTM165/166-48nt transgenic plants had defective flower, we chose STTM156/157 as the "male" parent for crossing since repression of miR156/157 did not show obvious phenotype in flower structure. Their resistance to hygromycin antibiotics and herbicide BASTA screened the hybrid seeds. The hybrid plants showed the similar phenotype of STTM156/157 with turbine-blade shaped cotyledons at early juvenile developmental stage (Fig. 2.2A). Interestingly, when the hybrid plants grew into the adult stage, the plants exhibited the phenotype of the STTM165/166-48nt plants, with twisted stem, 
true leaves losing the asymmetry, and strong anthocyanin accumulation in both leaves and stems (Fig. 2.2B). These observations not only indicated the success of production of the hybrid plants between STTM165/166 and STTM1566/157, but also suggested that overexpression of HD-ZIPIII by STTM165/166 can surpass the repressing role of SPLs, induced by STTM156/157, in anthocyanin production. 


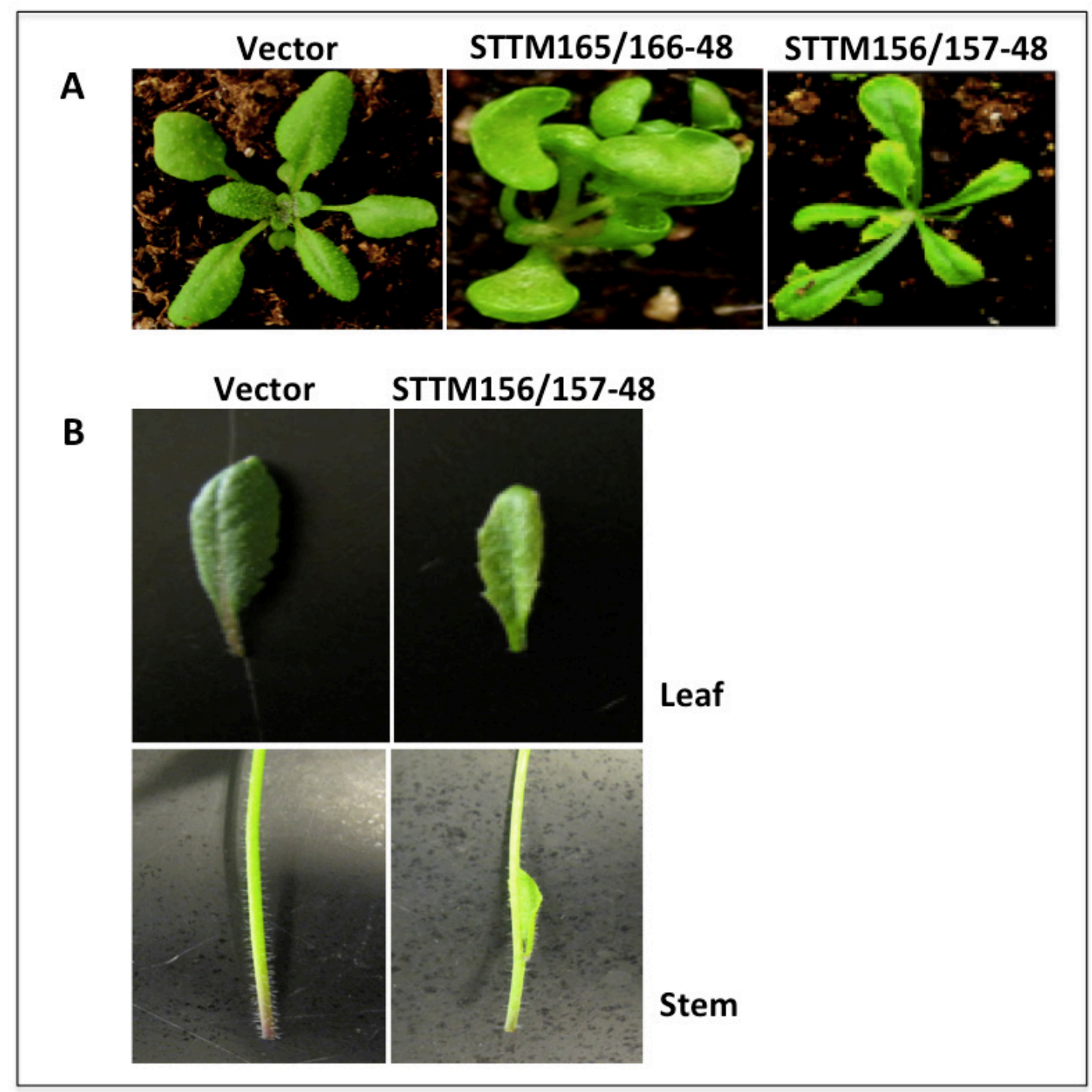

Figure 2.1 STTM165/166and STTM156/157 exhibit obvious phenotypes. (A) Phenotypes of 3-week-old seedlings of vector control, 1X35S-STTM165/166-48nt, and 2X35S-STTM156/157-48 transgenic plants. (B) Loss of the anthocyanin in STTM156/157 leaf and stem part compared with vector control at 5-week stage. 


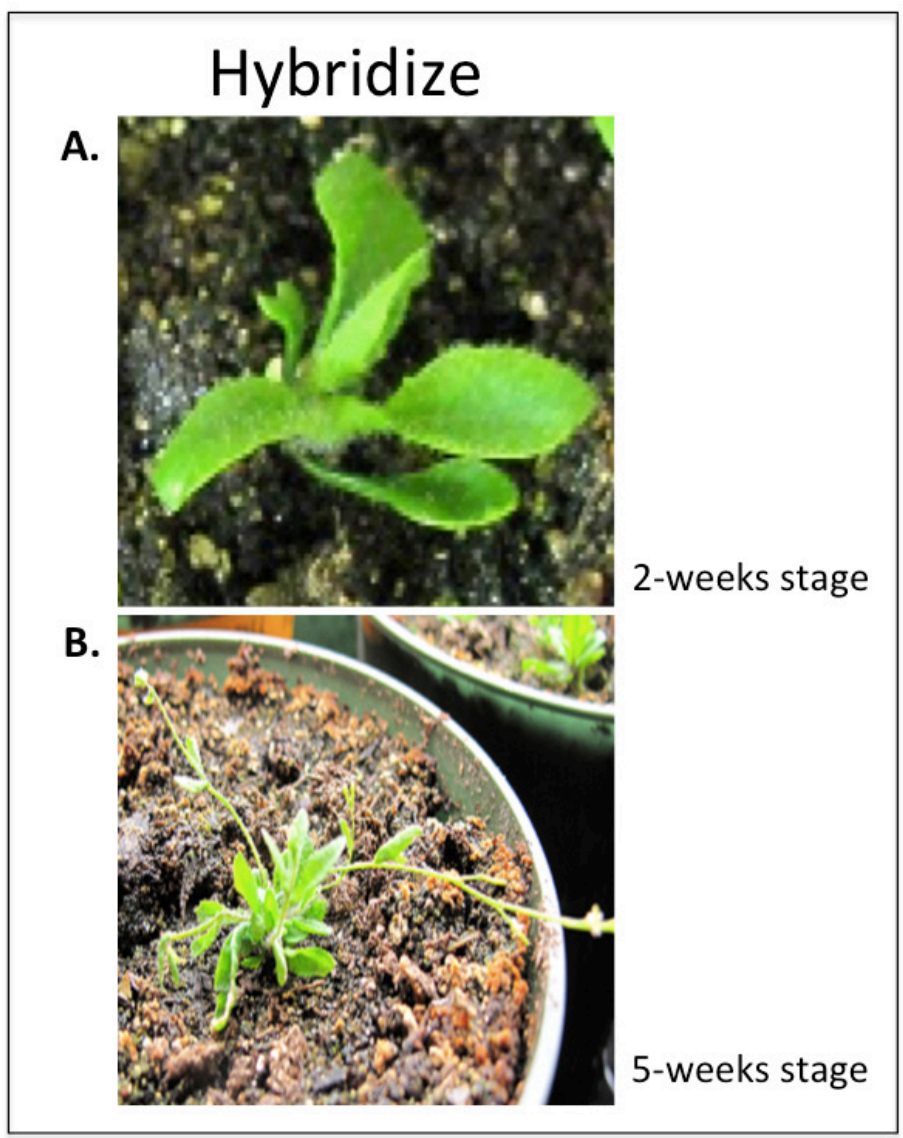

Figure 2.2. The phenotype of hybrid plant between STTM156/157 and STTM165/166. (A) 2-week stage hybrid plant; (B) 5-week stage hybrid plant.

\subsubsection{MiR165/166 and miR156/157 were simultaneously and functionally suppressed by STTMs}

Previous researches on miRNAs using STTM are primarily focused on individual miRNAs. To expand the application of 
STTMs to study the functional interaction between two or more miRNAs, we produced hybrid transgenic Arabidopsis by crossing STTM156/157 and STTM165/166. We conducted Northern blot analysis to detect the expressions of miR165/165 and miR156/157 in the hybrid plants. Both the miR165/166 and miR156/57 were dramatically reduced in the hybrid plant compared with empty vector control plant (Fig. 2.3A). We also checked the miR156/157 and miR165/166 expression level in the parent lines as controls. We further determined the expressions of the target genes of miR165/166 and miR156/157 in the hybrid plant, and the results showed HD-ZIP III family and SPL family genes were up-regulated significantly (Fig. 2.3B), especially for the miR156/157 target genes, as is correlated with the stronger promoter (2X35S) used for STTM156/157-48nt, as compared with the promoter (1X35S) for STTM165/166-48nt. To exclude the possibility that reduction of miR156/157 in the hybrid line might be due to the off-target effect of STTM165/166-48nt, we assayed the parent line of STTM165/166-48nt for the expression of miR156/157 and the result showed that expression of 
miR156/157 was not affected in the parent line of STTM165/166-48nt (Fig. 2.3A). Taken together, all these results demonstrated that we successfully blocked two miRNA families by STTMs using a crossing approach.

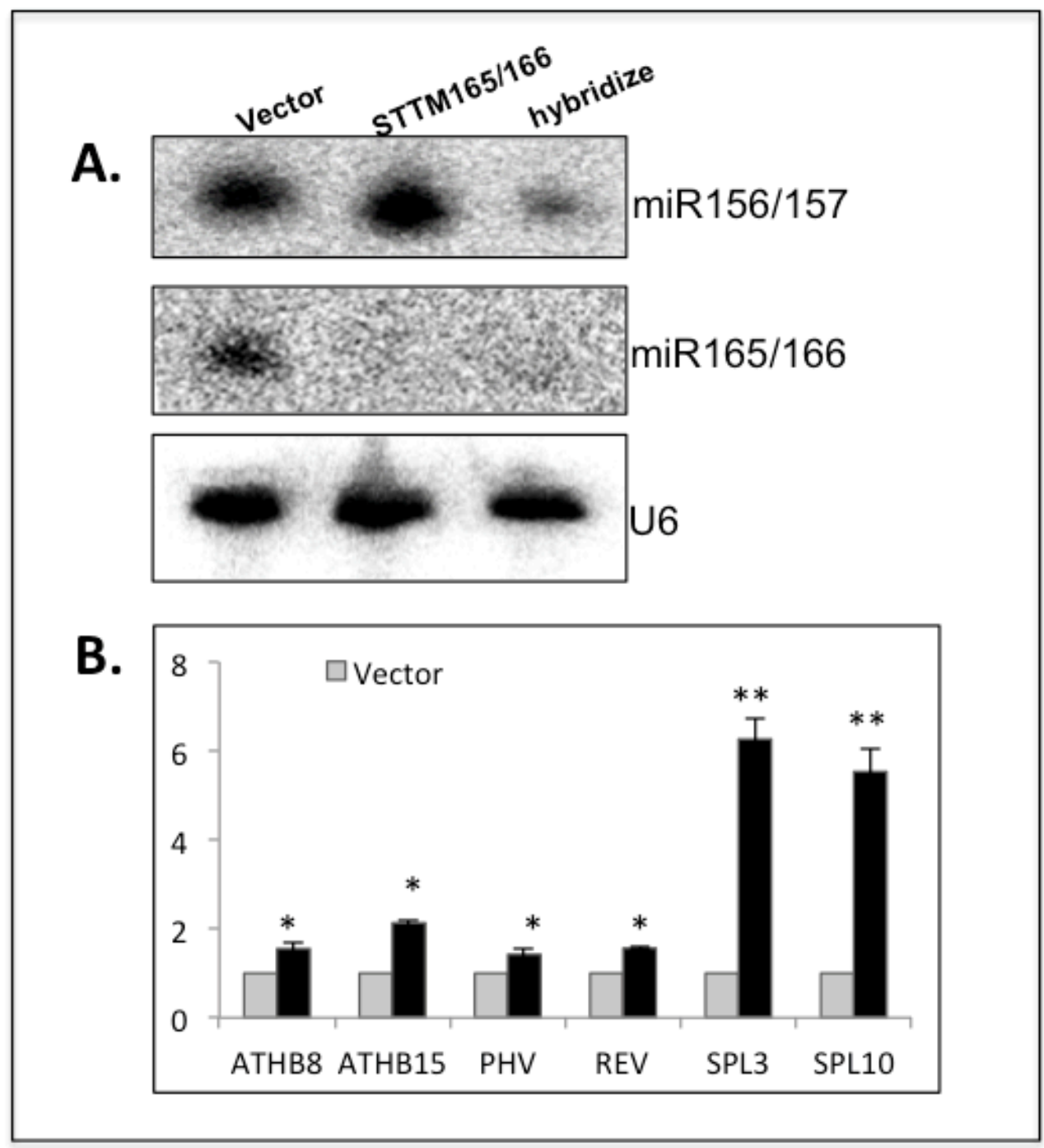

Figure 2.3. Detecting the expressions of miR165/166, miR156/157 and their target genes in the hybrid plant. (A) Northern blot result of miR165/166 and miR156/157 in the hybridplant. (B) The expressions of miR165/166 target gene HD-ZIP III family and miR156/157 target SPL family were detected by qRT-PCR. 
The total RNA was isolated from the hybrid plant leaves at 2-weeks-old seedling stage. Empty vector transgenic plant was used as a control. Actin2 was used for internal control. Bars show SE. ** with p-value $<0.01$ are statistically very significant and ${ }^{*}$ with $\mathrm{p}$-value $<0.05$ are statistically significant.

\subsubsection{MiR165/166 regulates anthocyanin predominantly in leaves, while miR156/157 regulates anthocyanin in both leaves and stems}

In the STTM165/166-48nt transgenic plants, the anthocyanin was up regulated in both the leaf and the stem (Fig. 2.4A; Fig.

2.4B). In contrast, anthocyanin is STTM156/157-48nt transgenic plants was down regulated in both positions (Fig. 2.4A; Fig. 2.4B). Yet, the anthocyanin was observed in the leaf but not the stem of the hybrid plant, suggesting a dominant effect of STTM165/166 on the anthocyanin accumulation over the STTM156/157 mainly in leaves of the hybrid line. The leaf size of the hybrid plant was similar to STTM165/166-48nt transgenic plant, and the anthocyanin was up regulated in the leaves of both the hybrid line and the parent STTM165/166 line, but not the STTM156/157 line. The observation that the 
anthocyanin was repressed in the stem of the hybrid line as compared with the vector control transgenic plants and STTM165/166-48nt line, together with the above observations, suggests that the effect of STTM165/166 suppressed the effect of STTM156/157 in anthocyanin production in leaves but not in stems. In summary, miR165/166 regulates the anthocyanin synthesis predominantly in the leaf organ over miR156/157, and miR156/157 regulates anthocyanin synthesis predominantly in the stem over miR 165/166.

We further did in situ hybridization and immune-histochemistry staining to check the miR165/166 and miR156/157 localization in leaf (Figure 2.5). The miR165/166 and miR156/157 signals were presented with Green, and the cell nucleus was localized with DAPI as presented with Blue. Based on in-situ immune-histo staining, the expressions of miR165/166 and miR156/157 were repressed significantly in the hybrid line as compared with empty vector transgenic plants. 


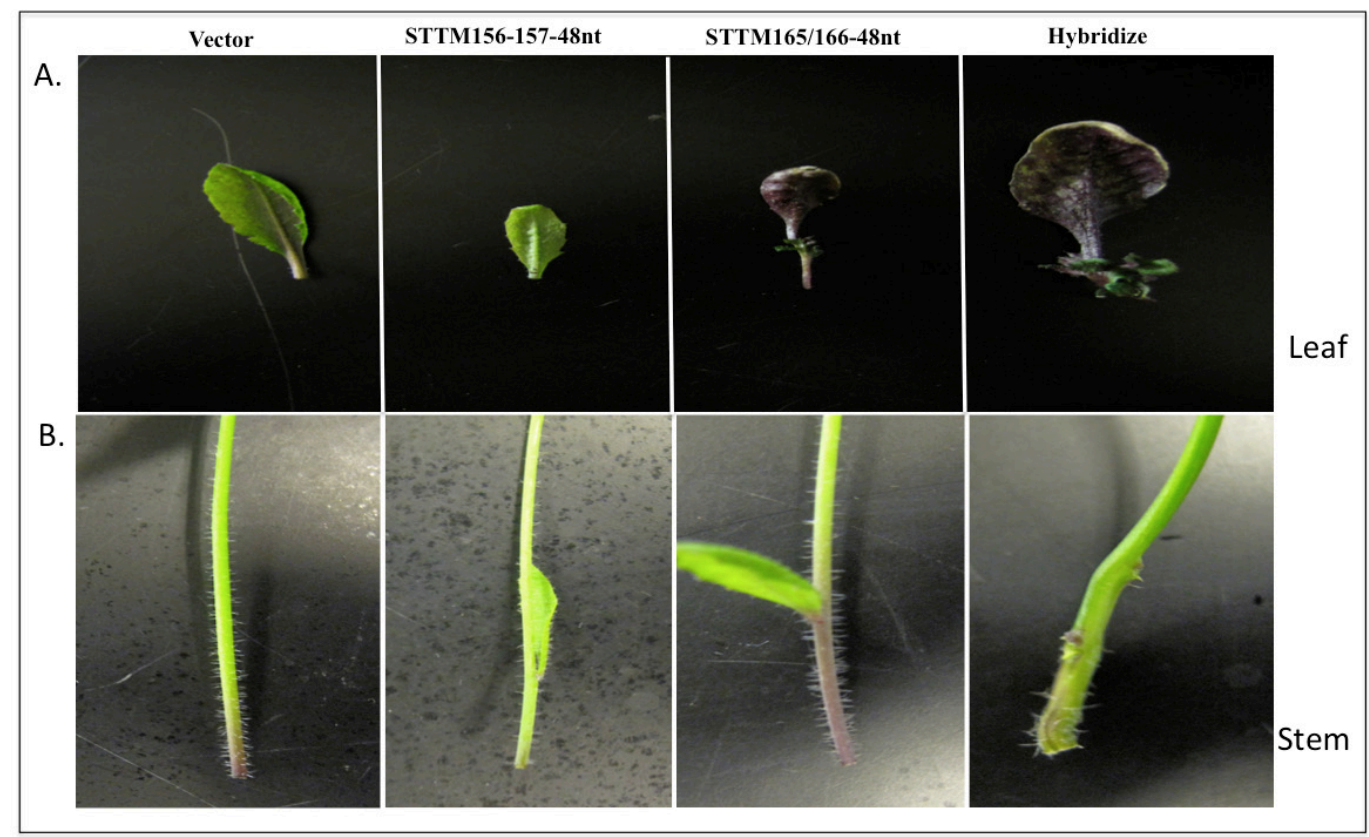

Figure 2.4. The anthocyanin accumulation in vector control, STTM156/157, STTM165/166, and the hybrid plant at 5-weeks stage. (A) The anthocyanin in leaves. (B) The anthocyanin in stems. 


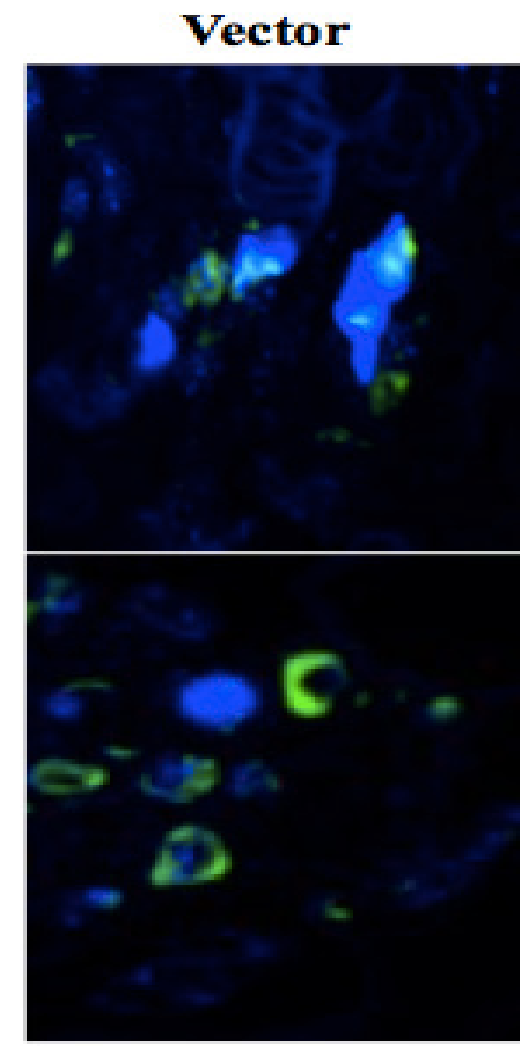

\section{Hybridization}

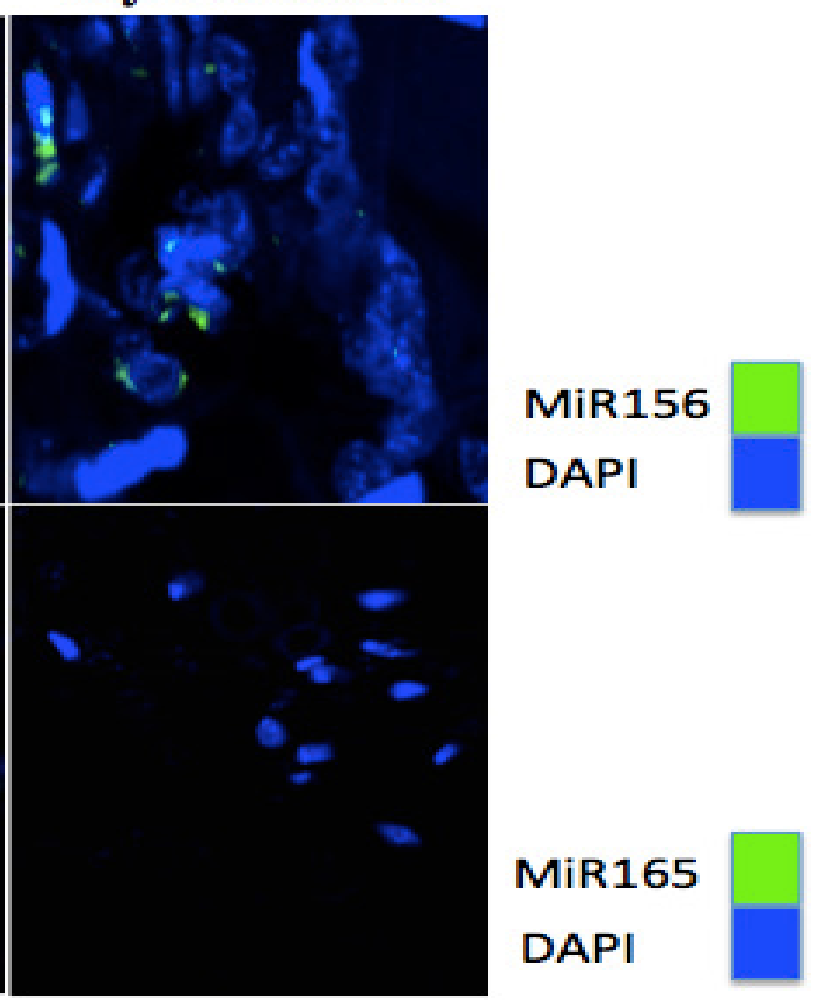

Figure 2.5. In situ hybridization and immunostaining of Arabidopsis leaf Sections. In situ hybridization was performed on leaf sections with miR165 probe or miR156 probe. The two-color overlay images showed that miR165 (green) and miR156 (green) was expressed low in the hybrid plant. The nuclei were labeled by DAPA (Blue). Empty vector used for control plant.

\subsection{Discussion}

The previous studies indicate that miR156/157-targeting gene SPL9 can negatively regulate the expression of 
dihydroflavonol 4- reductase (DFR), which is an important gene that induces the anthocyanin biosynthesis [88]. Since STTM repressed miR156/157 expression, in turn, the miR156/167 target gene SPL9 was up regulated, and high activity of SPL9, as a transcription repressor, then repressed DFR gene expression. This well explained the repression of anthocyanin in the STTM156/157-48nt transgenic plants.

Previous study also showed that anthocyanin pathway genes were up regulated in the STTM165/166-48nt transgenic plants, like F3H, DFR, ANS, and CHS [93], the underlying mechanism was unknown. Interestingly, the hybrid plants exhibited distinguished phenotype that is partially similar to either STTM156/157-48nt or STTM165/166 transgenic plants with turbine-blade shaped cotyledons like STTM56/157 phenotype and spoon-shaped cotyledons shown in STTM165/166-48nt transgenic plants at the early development stage. More interestingly, anthocyanin accumulation was observed in the stem of the hybrid plants before the juvenile stage, but not at the adult stage as compared with its parent 
lines, STTM165/166-48nt and STTM156/157-48nt, showing a sequential and dynamic change of anthocyanin accumulation in the leaves and stems of the hybrid line. Specifically in detail for this observation, I found that the hybrid plants had a high level of anthocyaninin stems before juvenile stage, and this high anthocyanin level was decreased dramatically when the hybrid plant was entering to the adult stage. In contrast, the anthocyanin level, low in the leaves of the hybrid before juvenile stage, increased significantly in the leaves at the adult stage. Also found at the adult stage, the stem starts to turn into twisted like STTM165/166. Taken together, all these data demonstrated that the miR165/166 and miR156/157 functionally interact with each other and both can be blocked by STTMs at the same time, but the blocking effect on anthocyanin accumulation in the hybrid plants are really in dynamic changes in both the stem and the leaf organs. Previous study indicate that miR156/157 can negatively regulate anthocyanin biosynthesis through TCP repressing DRF anthocyanin biosynthesis pathway gene, also, in the STTM165/166-48nt transgenic plants, anthocyanin 
biosynthesis pathway gene were up regulated, we did not find that the evidence for the mechanism how miR165/166 regulates anthocyanin biosynthesis directly or indirectly. All the data showed that anthocyanin regulation by miRNAs was complicated in plant different developmental stages, and the regulation is rather dynamic. These phenomena may suggest a complex interactive regulation between the target genes of $\operatorname{miR} 165 / 166$ and miR156/157, and miR156/157 and miR165/166 regulatory networks are complicated, which requires further investigation. In conclusion, miRNAs are important regulatory elements in plants; aberrant expression of miRNAs may induce disorders in plant metabolism, in which miRNAs may play a synergistic or antagonistic role in the secondary metabolic pathways or even in the primary metabolic pathways. 


\section{Investigating miR165/166 regulatory network to in Arabidopsis}

Yiyou Gu, Haiping Liu, Mengmeng Qiao, Jun Yan, Sachin Teotia, Guiliang Tang

The following chapter is part of the manuscript under preparation. 


\subsection{Introduction}

In plant system, most miRNAs target transcription factors for down regulation [94]. Transcription factor is a protein that control specific gene transcription based on the specific binding motif of the transcription factor recognizing the regulated gene sequence on the gene promoter region [95, 96]. Transcription factors work alone or work with other protein as a complex, to promoting or repressing the target gene transcription rate[97, 98]. A clearly defined transcription factor has at least one DNA-binding-domain (DBD) and a transcription-activating domain[99, 100]. Different transcription factors that have the same DBD can be defined as one class of transcription factor [101]. In plant system, one miRNA usually have several target genes, such as miR165/166, having five target genes. These five genes are defined as HD-zip III family transcription factors because they have the same DBD [102]. The regulatory mechanisms of plant processes by miRNAs are complicated. For example, flower 
timing can be regulated by two miRNAs, miR156 and miR172[103]. The plant develops from juvenile stage to adult flowering stage, along with an up-regulation of miR172 expression level and a down regulation of miR156/157 over the process. While miR156/157 family target SPL3 and SPL4 for down-regulation [103], miR172 negatively regulates AP2 that further represses ARF3 and ARF4 expression [20]. In tas3 or ago 7 mutant, high expression levels of SPL3 and SPL4 genes are detected together with a high level of ARF3 and ARF4 [104, 105]. In this case, up-regulation of SPL3 and SPL4 may be due to the direct or indirect repression of miR156 by ARF3 and ARF4. These complex miRNA regulatory networks may explain the complicated plant developmental processes such as plant flowering. When the plants turn to flowering stage, the miR172 expression level is up regulated, and high level of miR172 represses the target gene AP2 gene activity. While low activity of AP2 improves the activity of ARF3 and ARF4 that further activate SPL genes in plants. The flower timing sets a good example for exploring the miRNA regulatory network in plants. In viewing the complicated 
phenotypes were induced in STTM165/166, we hypothesized there must exist a complicated miRNA regulatory network in such transgenic plant. The functions of miR165/166 targets, HD-ZIP III family genes, have been well studied using loss-of-function alleles[13]. Loss function of REV causes defects in leaf, stem, and vascular development[106, 107]. Defect of ATHB8 showed no phenotype[108]. PHB and PHV have overlapping function with REV in embryogenesis[109]. Also, there has no report for the loss function of ATHB15. Loss-function alleles of single mutant, double mutant, and triple mutant of HD-ZIP III family members have been generated [110]. The phenotype of each allele has been well described. In this study, we analyzed $p h b-p h v$-cna triple mutant (ABRC), together with STTM165/166-48nt transgenic plants, and found that except the stem, leaf and root organ having pleiotropic phenotypes, the flower organ also established abnormal morphology. In plant reproductive development, three miRNA-TF pairs, miR167, miR319, and miR159 and their target TFs, were found interacting with each other to regulate the biogenesis process of these miRNAs [111]. The 
defection of these miRNAs induces phenotypic alterations in the flower organ [111]. We hypothesized that similar miRNA networks were disturbed when endogenous miR165/166 targeting HD-ZIP III transcription factors was altered by STTM. To prove our hypothesis, we generated small RNA libraries from STTM165/166 transgenic plant and the empty vector control and sent them for sequencing. Specifically, I generated the transgenic plants, Dr. Jun Yan did the small RNA sequence work, and Dr. Guiliang Tang did the experiment design and data analysis. We found that miR319 was significantly up regulated in the STTM165/166-48nt transgenic plants. This observation suggests that miR319 may have direct or indirect regulatory relationship with miR165/166, which has not been reported by previous research.

\subsection{Experiment procedures}

\section{Plant lines and growth conditions}


All plant materials used in the experiments were performed in Arabidopsis thaliana or their mutant lines. 2X35S-STTM165/166-48nt has a herbicide BASTA selection marker. The T-DNA insertion mutant lines, CS6961 (rev-6), CS6964 (phb-12), CS6965 (phb-13), CS6966 (phv-11), CS6968 (cna-2), and CS6970 (athb8-12) were obtained from the Nottingham Arabidopsis Stock Centre, the triple mutant phb-phv-cna was obtained from hybridization using phb-13, $p h v-11$, and $c n a-2$ as a gift from Dr. Steven E. Clark's lab [112]. The phenotype of each mutant line has been described previously [112]. Transgenic seeds of homozygous STTM165/166-48nt plants were used for the experiments, and the empty vector transgenic plants used as the control. The seeds were first treated with $10 \%$ bleach for $10 \mathrm{~min}$, and followed with $1 \mathrm{~min} 70 \%$ ethanol sterilization. The surface sterilized seeds were washed with sterilized dd-water for three times. The seeds were first planted with $0.5 \mathrm{X}$ Murashige and Skong (MS) media on Petri dishes. After planting the seeds on Petri dishes, the plates were sealed with Parafilm to prevent contamination and desiccation. The dishes were cold treated at 
$4^{\circ} \mathrm{C}$ for 3 days and then exposed with $16 \mathrm{~h} \mathrm{light} / 8 \mathrm{~h}$ dark and $25^{\circ} \mathrm{C}$ conditions for seeds germination. The plants were transferred to the soil from Petro dishes to grow when the plants had four leaves. The transgenic plants 2x35S-STTM165/166-48nt were selected with BASTA twice after the plants were transferred from the Petro dishes to soil.

\section{Small RNA gel blotting}

Total RNAs were extracted from leaves of the wild-type, empty vector transgenic control, STTM165/166-48nt, and T-DNA insertion mutants using trizol (Invitrogen). Concentration of isolated total RNAs was measured with NANO-drop 2000 (Thermo Scientific). $10 \mu \mathrm{g}$ total RNA from each sample was fractionated on a 15\% PAGE under denaturing conditions. The $\mathrm{P}^{32}$ labeled probes complementary to sequence of the small RNAs were used for hybridization with Blots[113]. After PAGE gel electrophoresis, the gels were pre-soaked in 0.5X TBE buffer, and then the total RNAs were transferred from the gel to membrane using semi-dry gel 
transferring aparatus. The RNAs on the membrane were cross-linked by a UV Stratalinker 1800 . The membrane was pre-hybridized with pre-hybridization buffer at $37^{\circ} \mathrm{C}$ before adding the probe to continue hybridization overnight at $37^{\circ} \mathrm{C}$. The hybridized membrane was washed three times with washing buffer for 20 minutes each. The washed membrane was wrapped with saran and exposed to a phosphor-image plate overnight. The phosphor-image plate was then scanned with Scanner Typhoon 9410 and quantified by ImageQuant TL1 Software. U6 expression was detected as internal control. The probes sequences are listed in Table 3 .

\section{qRT-PCR}

The total RNA was isolated from 2-weeks-old seedling leaves using Trizol. The expression levels of pri-miRNA and miRNA targets were detected by qRT-PCR. We performed DNase treatment to the purified total RNAs to remove potential genomic DNA contaminations before used for reverse transcription. The random primer was used as the reverse 
transcription primer. qRT-PCR using the SYBR green PCR master mix kit was performed with an Applied Biosystems step one instrument (ABI). Actin mRNA was used as internal control. The sequences of primers used for qRT-PCR are listed in Table 2. The relative levels of gene expression were calculated using $2-^{\wedge}$ cycle threshold method[114]. Three biological replicates experiments were applied to ensure the reliable results.

\section{Small RNA deep sequencing}

This experiment was done by Jun Yan. Briefly, the total RNA was isolated from the leaves of 3-week-old seedlings. The small RNA was size fractionated using a polyacrylamide/urea gel electrophoresis. The small RNAs were purified from the gel, followed by ligating to a 3' Solexa DNA adaptor. The 3' DNA adaptor ligation product was purified before ligated to a 5' Solexa RNA adaptor[115]. The final small RNA libraries were obtained by a reverse transcription. Small RNA sequencing was conducted using Genome Analyzer II 
(Illumina) as previous described [115].

\section{In-situ \\ hybridization \\ and immunohistochemistry staining}

Dissected leaves from STTM165/166-48nt transgenic plant and the empty vector control were fixed in $4 \%$ formaldehyde (pH 7.4) overnight at $4^{\circ} \mathrm{C}$ and then processed routinely for paraffin embedding. Tissues were cut into $10 \mu \mathrm{m}$ sections and placed to glass slides (Superflost, Fisher Scientific). For in-situ hybridization, sections were first deparaffinized and rehydrated, then treated with Proteinase K (Roch, $40 \mu \mathrm{g} / \mathrm{ml}$ ) as described [92]. Briefly, a total of 3 pmol of DIG-labeled Locked Nucleic Acid (LNA) probes (Exiqon) were mixed with $200 \mu \mathrm{l}$ of hybridization buffer and applied onto the slides in order to hybridize at $37^{\circ} \mathrm{C}$ for overnight. Slides were then washed using 2X SSC (saline-sodium citrate) solution and incubated with alkaline phosphatase-conjugated sheep anti-DIG antibody (Roche) at $4{ }^{\circ} \mathrm{C}$ for overnight. Alkaline phosphatase reaction was carried out with $50 \mathrm{mg} / \mathrm{ml}$ of NBT/BCIP staining solution 
at room temperature for 1-3 days. The images were captured on Olympus FluoView FV1000 confocal microscopy.

\section{RNA deep sequencing}

Sachin Teotia did this experiment. Total RNAs were isolated from leaves of the STTM165/166-48nt transgenic plants and empty vector at 3 weeks old. The RNA quality was assayed for its RNA integrity number (RIN) using a Bio-analyzer. cDNA libraries were constructed by University of Michigan DNA sequencing core facility. The resultant libraries were sequenced using Illumina HiSeq platform. The expression levels of miRNAs and mRNAs were then analyzed after RNA-seq and small RNA-seq.

\section{Analysis for HD-ZIP III transcription factor binding motifs through BLASTN using the conserved nucleotide motif}

The promoter sequence of miRNA was obtained from National 
Center for Biotechnology Information (NCBI). We defined the 5000 nucleotides before the miRNA stem-loop as the miRNA promoter region. The promoter sequence was saved in SnapGene Viewer. The published HD-ZIP III transcription factor binding motifs, TAAATG[C/T]A and GTAAT[G/C]ATTAC, were used for BLASTN analysis with an allowance of one mismatch[116, 117].

\section{Statistical analysis}

All results are expressed as mean $\pm \mathrm{SD}$. Statistical significance is determined by unpaired Student's t-test (two-tailed) and ANOVA analysis. Each gene expression was checked at least for three independent experiments.

\subsection{Results}




\subsubsection{Repression of miR165/166 by STTM altered the expressions of other miRNAs}

The repression of miR165/166 by STTM induced dramatic phenotypic changescompared with the empty vector control plants (Fig. 3.1A). The strong phenotype of STTM165/166 transgenic plants may be due to abnormal expressions of a wide genes. To fully understand miR165/166 functions and the potential the miRNA regulatory network related to miR165/166, we preformed small RNA deep sequencing to analyze the small RNA expression pattern in the STTM165/166 transgenic plants. Total RNAs were isolated from the 3-weeks-old seedlings. We applied the RNA sample for small RNA gel blotting to confirm the repression of miR165/166 in transgenic plant before sending the materials for small RNA deep sequencing. The Northern blot result showed that the miR165/166 were decreased dramatically in the transgenic plant (Fig. 3.1B). The small RNA sequencing result indicated that several miRNAs exhibited abnormal expressions compared with the vector control plant. 
Particularly, miR319 was up regulated to about 8 times in STTM165/166 transgenic plant compared to the control (Fig

\subsection{C).}

MiR165/166 target gene HD-zip III family was up regulated in the STTM165/166 transgenic line. Because HD-zip III family members are important transcription factors in control of plant development, the up-regulated miR319 may be controlled by HD-ZIP III directly or indirectly. What is more, previous study indicated that highly expressed miR319 induced a strong crinkle and twisted leaf phenotype in tomato leaf morphology [118]. Indeed, we also observed a similar phenotype in STTM165/166 transgenic plants. This indicates that the partial phenotype that exhibited in STTM165/166 may be due to the up-regulation of miR319.

We further validated miR319 expression of the small RNA deep sequence result using q-RT PCR. Total RNAs were isolated from 3-week-stage seedlings for q-PCR analysis. MiR319 was increased significantly in the STTM165/166 
transgenic plant compared with the vector control (Fig. 3.2A). We also assayed for miR319 expression and localization in the leaf by in-situ immunohistochemistry staining, and the results also confirmed the high expression of miR319 in STTM165/166 compared with the control (Fig. 3.2B).

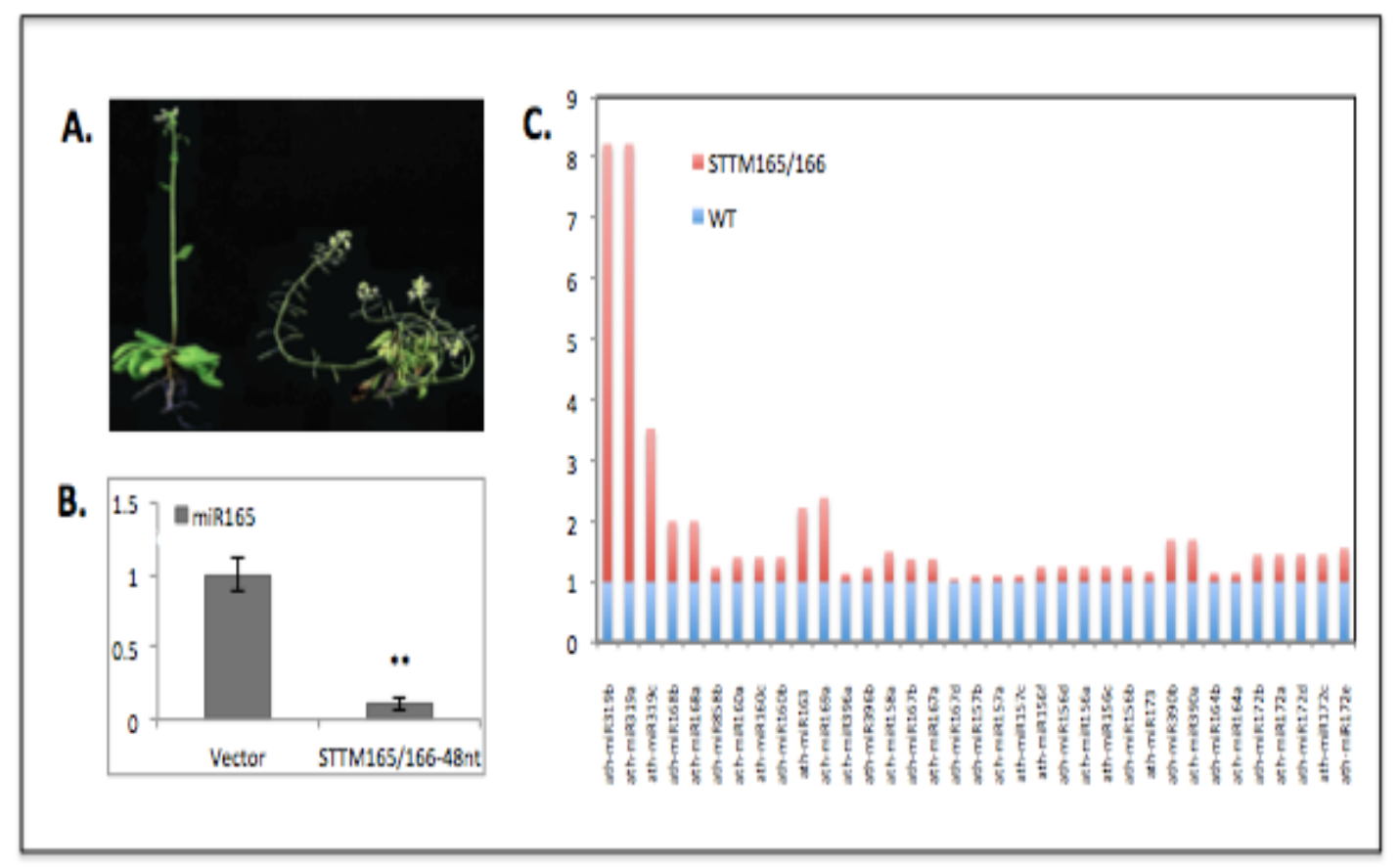

Figure 3.1 Small RNA deep sequencing of STTM165/166-48nt. (A) The STTM165/16648nt transgenic plant exhibits strong phenotype compared with the empty vector control. (B) The total RNA were isolated in the 3-weeks-old leaves. qRT-PCR was assayed to detect miR165/166 in the STTM165/166-48nt transgenic plant and the control. Data are shown as mean $\pm \mathrm{SD}$. ${ }^{* *}$ with $\mathrm{p}$-value $<0.01$ are statistically very significant and * with p-value $<0.05$ are statistically significant. (C) The miR319b and 319a were up-regulated revealed by small RNA sequencing result. 
The small RNA reads number in STTM165/166 was normalized to the reads in vector control. The red line indicates the small RNA expression in STTM165/166-48nt, and the blue indicates the small RNA expression in the vector control.

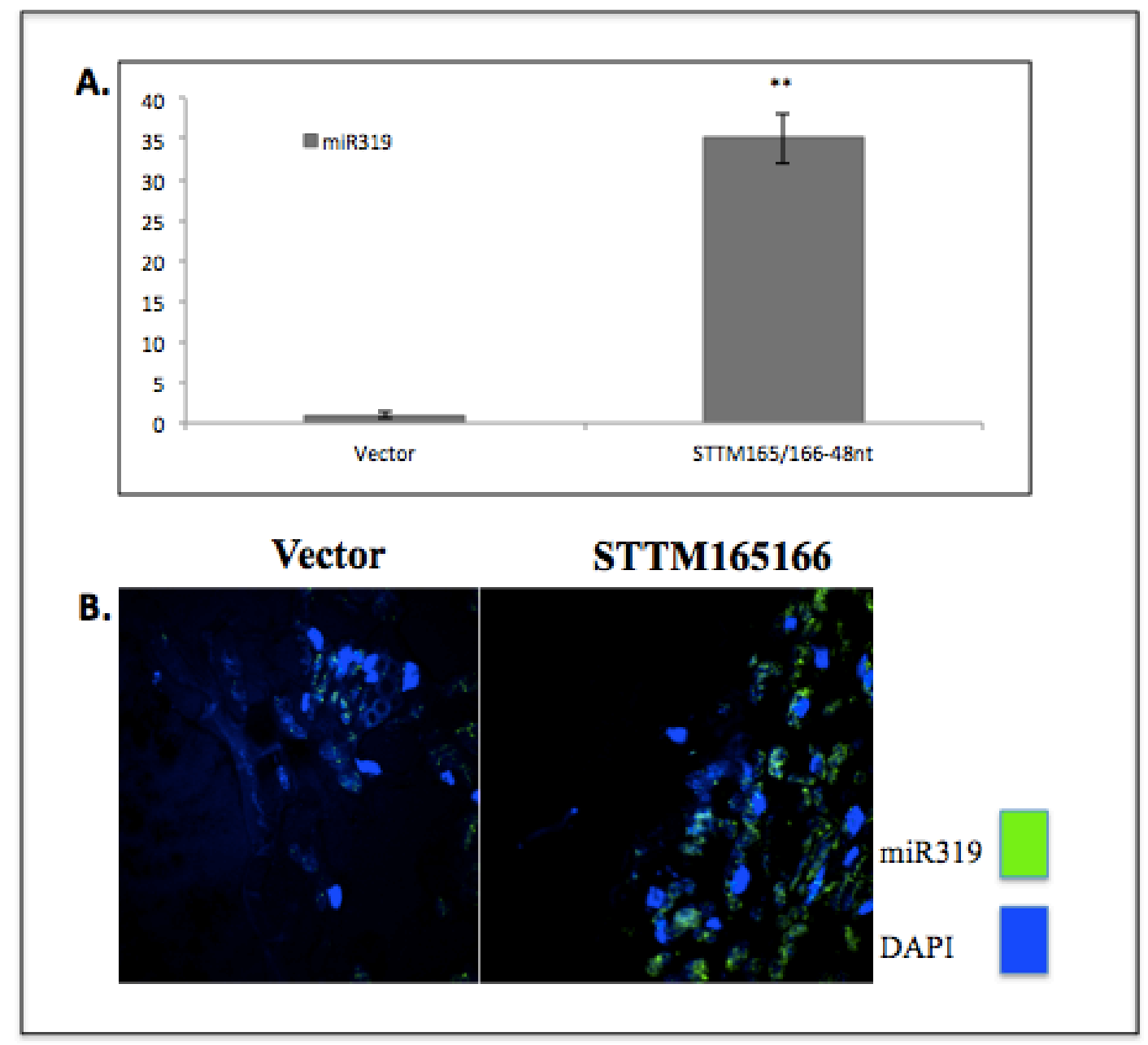

Figure 3.2 Detection of miR319 expression level in STTM165/166-48nt transgenic and the control plants. (A) Detection of miR319 expression by qRT-PCR. (B) The expression and localization of miR319 in Arabidopsis leaves using in-situ immunohistochemistry staining. The nucleus was labeled by DAPA (Blue), and miR319 was labeled by miR319 probe (green). Data are shown as mean \pm SD. ** with $\mathrm{p}$-value $<0.01$ are statistically very significant and * with $\mathrm{p}$-value $<0.05$ are statistically significant. 


\subsubsection{The phb-phv-cna triple mutant expressed more miR319 but repressed the REV expression}

Previous results showed that miR319 was up regulated in STTM165/166-48nt transgenic plants. MiR165/166 target HD-ZIP III family genes may play an important role in regulating miR319 expression. To better understand the regulatory network between miR165/166 and miR319, we obtained the PHB, PHV and CNA triple genetic mutant seeds (phb-phv-cna) from Dr. Steven E. Clark's lab for analysis. The triple genetic mutant plant exhibited strong phenotype compared with the wild-type control plant (Fig. 3.3A). Since the highly activated HD-ZIP III promoted miR319 expression in STTM165/166 transgenic plant, we thought that phb-phv-cna might repress miR319 expression. But the result was not as what we expected; miR319 was also up regulated significantly in phb-phv-cna plant (Fig. 3.3B). This result suggests that HD-ZIP III might not just simply promote miR319 expression, but some HD-ZIP III family members may 
also repress miR319 expression. Previous study also indicated that the HD-ZIP III family genes have overlapping, antagonistic roles in Arabidopsis development [110]. We also detected the other two family members ATHB8 and REV for their expression in phb-phv-cna mutant line. There was observed no big difference in ATHB8 expression between the triple mutant and the control (Fig. 3.3C), but REV was repressed in the triple mutant significantly by roughly 3 times (Fig. 3.3C). We found that miR319 was up regulated significantly in phb-phv-cna line by about 3 times. These results indicated that HD-ZIP III could regulate miR319 expression in a complicated manner. While some members may repress miR319 expression other members can promote miR319 expression. 


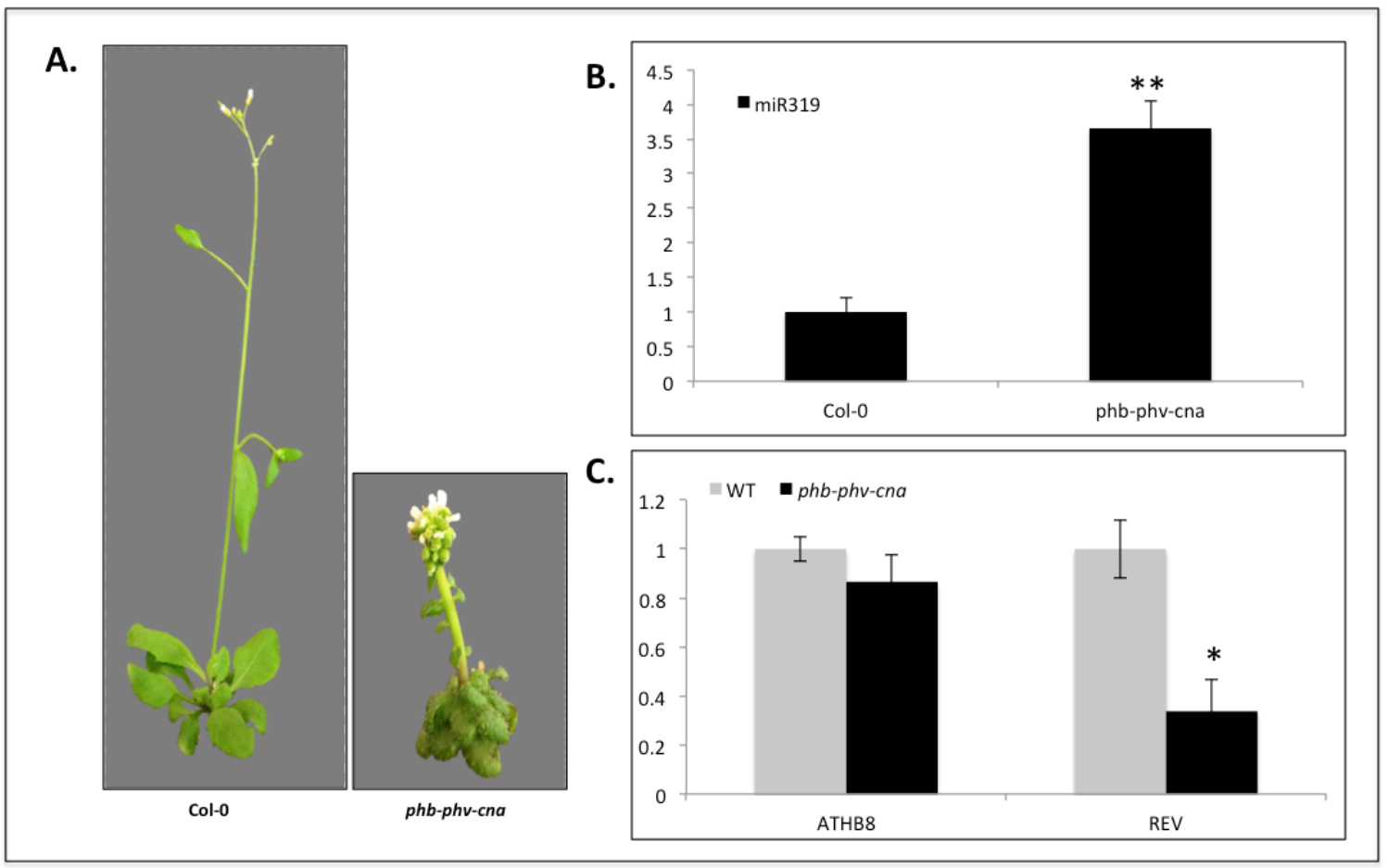

Figure 3.3 The expressions of miR319, ATHB8 and REV in phb-phv-cna triple mutant. (A) The plant phenotype of phb-phv-cna genetic mutant plant. (B) The miR319 expression in phb-phv-cna genetic mutant plant. (C) ATHB8 and REV expressions in $p h b-p h v$-cna genetic mutant plant. Data are shown as mean $\pm \mathrm{SD}$. ** with $\mathrm{p}$-value $<0.01$ are statistically very significant and * with p-value $<0.05$ are statistically significant.

\subsubsection{MiR319 expression in HD-ZIP III single genetic mutant plants}

The HD-ZIP III transcription factors include five members, ATHB8, ATHB15, PHB, PHV, and can [119]. To further 
understand the regulation mechanism between miR319 and miR165/166, we ordered the HD-ZIP III family T-DNA insertion mutant seeds of CS6961 (rev), CS6964 (phb), CS6966 (phv), CS6968 (athb15), and CS6970 (athb8) from ABRC. The phenotype of each line were well described [112]. We detected miR319 expression level in each genetic mutant plant using qRT-PCR. The results showed that miR319 was up regulated in rev, athb8, and athb15 mutant plant significantly (Fig. 3.4A, Fig. 3.4B, Fig. 3.4C), while there have no difference of miR319 in phb and phv mutant plants compared to the control (Fig. 3.4D, Fig.3.4E). These results suggest that ATHB8, ATHB15, and REV negatively regulate miR319 expression either directly or indirectly. All five HD-ZIP III family members were up regulated due to the repression of miR165/166. Since REV, ATHB8, and ATHB15 negatively regulate miR319 expression, the upregulated miR319 may be promoted by PHB and PHV. We didn't detect any difference of miR319 in PHB or PHV single mutant, which means that PHB and PHV should have overlapping regulatory functions on miR319. 


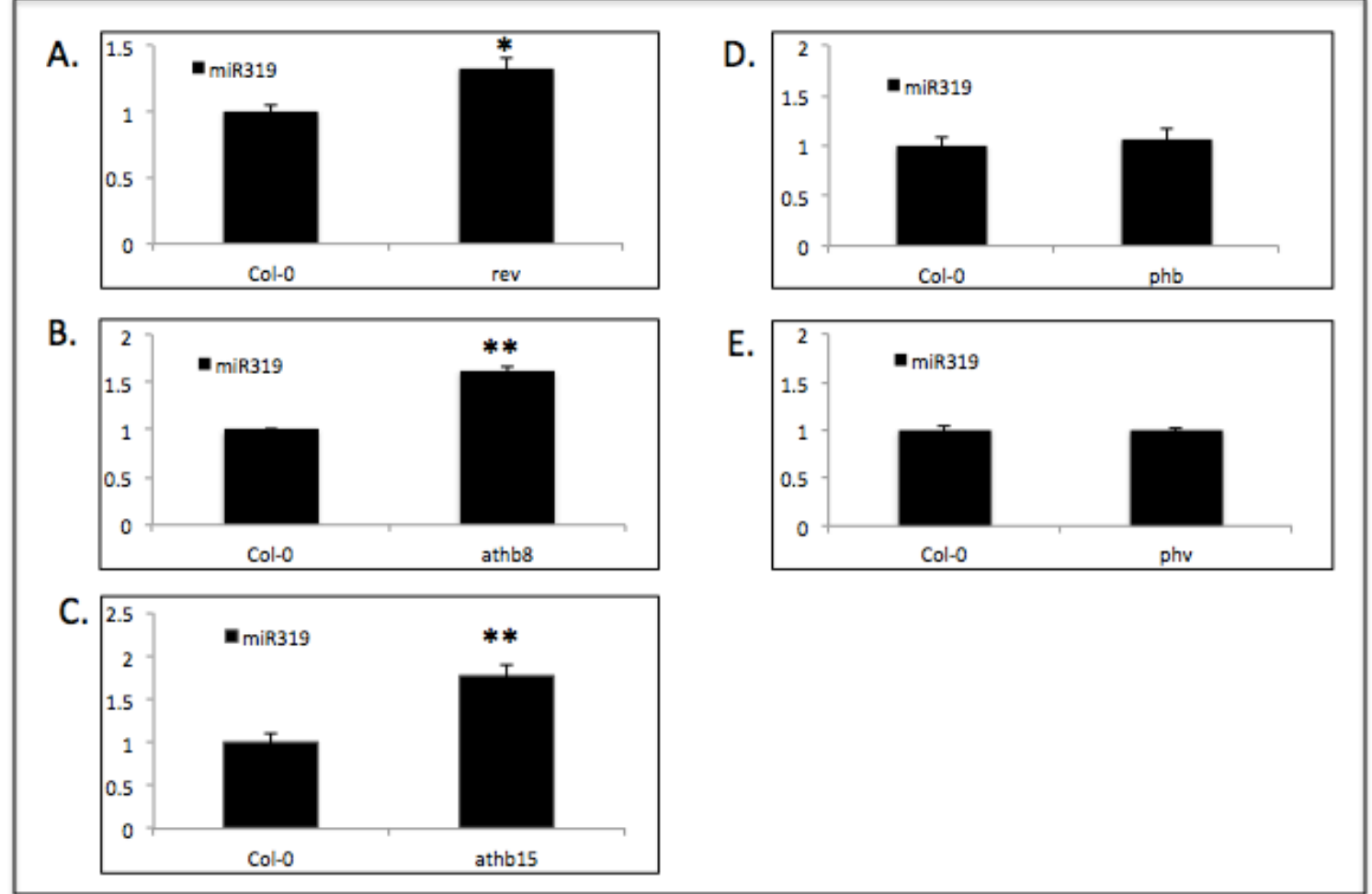

Figure 3.4 The miR319 expressions in HD-ZIP III single genetic mutant and the control plants. (A) MiR319 expression in REV genetic mutant plant. (B) MiR319 expression in ATHB8 genetic mutant plant. (C) MiR319 expression in ATHB15 genetic mutant plant. (D) MiR319 expression in PHB genetic mutant plant. (E) MiR319 expression in PHV genetic mutant plant. Data are shown as mean $\pm \mathrm{SD}$. ** with $\mathrm{p}$-value $<0.01$ are statistically very significant and * with p-value $<0.05$ are statistically significant.

\subsection{Discussion}


In the STTM165/166-48nt transgenic plants, STTM transcripts repressed miR165/166 dramatically. The STTM165/166 transgenic plants exhibited strong phenotypes. The previous research indicated that HD-ZIP III family genes play a role in the shoot and root apical meristem, also functions on vascular meristems[120]. HD-ZIP III family genes have functions in meristem initiation, leaf development in a complex manner with overlapping and antagonistic roles[121]. Like that REV, PHB, and PHV are close related, with overlapping functions in the post-embryonic shoot, ATHB8 and ATHB15 may have antagonizing functions in the bioprocessing of miR319n [122]. We detected ATHB8 and REV in phb-phv-cna triple mutant line with no expressional differences as compared to the control, but REV was repressed significantly in this triple mutant, which means that HD-ZIP III might have self-regulatory function in control the expression of its family members. The binding motif of HD-ZIP III transcription factors are: TAAATG[C/T]A, GTAAT[G/C]ATTAC[123]. We found that this binding motif existed in REV but not the ATHB8 promoter region, which might suggest why REV, but 
not ATHB8, was repressed in phb-phv-cna mutant plant. The dynamic regulatory mechanism of HD-ZIP III family may play an important role in fulfilling the function of HD-ZIP III transcription factor.

MiR319 was up regulated in STTM165/166-48nt transgenic plants as well as in phb-phv-cna triple mutant line. Taking all that results together, we concluded that the five HD-ZIP III family members can differentially regulate the expression of miR319, as we observed that either high-expression of HD-ZIP III or low-expression HD-ZIP III can promote the transcription of miR319 genes. HD-ZIP III transcription factor binding motif also exists in miR319 gene promoter. This indicated that HD-ZIP III could regulate miR319 expression directly with a bidirectional characteristic. MiR319 further regulated TCPs, playing a important role in plant senescence, as demonstrated by the fact that low level of TCPs caused a delay in senescence and high level of TCP4 caused premature of plant [38]. In STTM165/166-48nt, four HD-ZIP family members were significantly up regulated, but ATHB8 expression level 
remains normal compared with other four members. In phb-phv-cna mutant line, REV was repressed significantly, but ATHB8 remains normal, just like its expression in STTM165/166-48nt transgenic line. The expression of ATHB8 was not affected by the mutation of other four HD-ZIP III members. We hypothesized that there has a network between PHB, ATHB15, PHV, and REV, and these proteins could promote or repress miR319 gene transcription. The altered expressions of HD-ZIP III gene can promote or repress miR319 expression.

Taken all the results together, we hypothesized that PHB, PHV are possibly the transcriptional activators, while HB8, CNA, and REV are likely the transcriptional repressors. Finally, we defined the regulatory networks between miR165/166 and miR319 as the following pattern in Figure 3.5. 


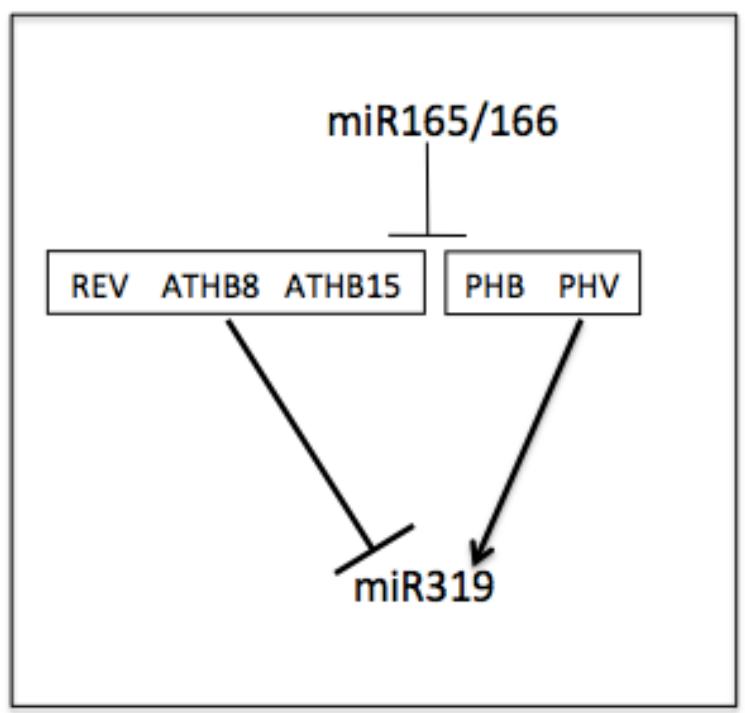

Figure 3.5. The regulatory networks between miR165/166 and miR319. 


\section{Reference}

1. Eckardt, N.A., The plant cell reviews aspects of microRNA and PhasiRNA regulatory function. Plant Cell, 2013. 25(7): p. 2382.

2. Ni, J.Z., E. Chen, and S.G. Gu, Complex coding of endogenous siRNA, transcriptional silencing and H3K9 methylation on native targets of germline nuclear RNAi in C. elegans. BMC Genomics, 2014. 15: p. 1157.

3. Budak, H. and M. Kantar, Harnessing NGS and Big Data Optimally: Comparison of miRNA Prediction from Assembled versus Non-assembled Sequencing Data--The Case of the Grass Aegilops tauschii Complex Genome. OMICS, 2015. 19(7): p. 407-15.

4. $\quad \mathrm{Li}, \mathrm{J}$. and A.A. Millar, Expression of a microRNA-resistant target transgene misrepresents the functional significance of the endogenous microRNA: target gene relationship. Mol Plant, 2013. 6(2): p. 577-80.

5. Franco-Zorrilla, J.M., et al., Target mimicry provides a new mechanism for regulation of microRNA activity. Nat Genet, 2007. 39(8): p. 1033-7.

6. Meng, Y., et al., Target mimics: an embedded layer of microRNA-involved gene regulatory networks in plants. BMC Genomics, 2012. 13: p. 197.

7. Shen, J., et al., Phosphorus dynamics: from soil to plant. Plant Physiol, 2011. 156(3): p. 997-1005.

8. Kuo, H.F. and T.J. Chiou, The role of microRNAs in phosphorus deficiency signaling. Plant Physiol, 2011. 156(3): p. 1016-24.

9. Pacak, A., et al., Heat Stress Affects Pi-related Genes Expression and Inorganic Phosphate Deposition/Accumulation in Barley. Front Plant Sci, 2016. 7: p. 926.

10. Cao, D., et al., Regulations on growth and development in tomato cotyledon, flower and fruit via destruction of miR396 with short tandem target mimic. Plant Sci, 2016. 247: p. $1-12$.

11. Jia, X., et al., Functional plasticity of miR165/166 in plant development revealed by small tandem target mimic. Plant Sci, 2015. 233: p. 11-21.

12. Zhang, $\mathrm{H}_{\text {., }}$ et al., Short tandem target mimic rice lines uncover functions of miRNAs in regulating important agronomic traits. Proc Natl Acad Sci U S A, 2017. 114(20): p. 5277-5282.

13. $\mathrm{Du}, \mathrm{Q}$. and $\mathrm{H}$. Wang, The role of HD-ZIP III transcription factors and miR165/166 in vascular development and secondary cell wall formation. Plant Signal Behav, 2015. 10(10): p. e1078955.

14. Xu, M., et al., Developmental Functions of miR156-Regulated SQUAMOSA PROMOTER BINDING PROTEIN-LIKE (SPL) Genes in Arabidopsis thaliana. PLoS Genet, 2016. 12(8): p. e1006263. 
15. Schommer, C., et al., Repression of cell proliferation by miR319-regulated TCP4. Mol Plant, 2014. 7(10): p. 1533-44.

16. Rhoades, M.W., et al., Prediction of plant microRNA targets. Cell, 2002. 110(4): p. 513-520.

17. Chen, M., et al., Functional characterization of plant small RNAs based on next-generation sequencing data. Comput Biol Chem, 2010. 34(5-6): p. 308-12.

18. Rubio-Somoza, I., et al., Regulation and functional specialization of small RNA-target nodes during plant development. Curr Opin Plant Biol, 2009. 12(5): p. 622-7.

19. Li, S., et al., HYL1 controls the miR156-mediated juvenile phase of vegetative growth. J Exp Bot, 2012. 63(7): p. 2787-98.

20. Spanudakis, E. and S. Jackson, The role of microRNAs in the control of flowering time. J Exp Bot, 2014. 65(2): p. 365-80.

21. Yaish, M.W., J. Colasanti, and S.J. Rothstein, The role of epigenetic processes in controlling flowering time in plants exposed to stress. J Exp Bot, 2011. 62(11): p. 3727-35.

22. Hyun, Y., R. Richter, and G. Coupland, Competence to Flower: Age-Controlled Sensitivity to Environmental Cues. Plant Physiol, 2017. 173(1): p. 36-46.

23. Poethig, R.S., Vegetative phase change and shoot maturation in plants. Curr Top Dev Biol, 2013. 105: p. 125-52.

24. Licausi, F., M. Ohme-Takagi, and P. Perata, APETALA2/Ethylene Responsive Factor (AP2/ERF) transcription factors: mediators of stress responses and developmental programs. New Phytol, 2013. 199(3): p. 639-49.

25. Yant, L., et al., Orchestration of the Floral Transition and Floral Development in Arabidopsis by the Bifunctional Transcription Factor APETALA2. Plant Cell, 2010. 22(7): p. 2156-2170.

26. Tsaftaris, A., et al., Sequence Characterization and Expression Analysis of Three APETALA2-like Genes from Saffron Crocus. Plant Molecular Biology Reporter, 2012. 30(2): p. 443-452.

27. Zhang, C., et al., Cascading cis-cleavage on transcript from trans-acting siRNA-producing locus 3. Int J Mol Sci, 2013. 14(7): p. 14689-99.

28. Yan, J., et al., The REDUCED LEAFLET Genes Encode Key Components of the trans-Acting Small Interfering RNA Pathway and Regulate Compound Leaf and Flower Development in Lotus japonicus. Plant Physiology, 2010. 152(2): p. 797-807.

29. Garcia, D., et al., Specification of leaf polarity in Arabidopsis via the trans-acting siRNA pathway. Curr Biol, 2006. 16(9): p. 933-8.

30. Zhou, Y., et al., Spatiotemporal sequestration of miR165/166 by Arabidopsis Argonaute10 promotes shoot apical meristem maintenance. Cell Rep, 2015. 10(11): p. 1819-27.

31. Lin, P.C., et al., Identification of miRNAs and Their Targets in the Liverwort Marchantia polymorpha by Integrating RNA-Seq and Degradome Analyses. Plant Cell Physiol, 2016. 57(2): p. 339-58.

32. Nakata, M. and K. Okada, The Leaf Adaxial-Abaxial Boundary and Lamina Growth. Plants 
(Basel), 2013.2(2): p. 174-202.

33. Yan, J., et al., Effective small RNA destruction by the expression of a short tandem target mimic in Arabidopsis. Plant Cell, 2012. 24(2): p. 415-27.

34. Zhu, Y., et al., PtrHB7, a class III HD-Zip gene, plays a critical role in regulation of vascular cambium differentiation in Populus. Mol Plant, 2013. 6(4): p. 1331-43.

35. Zhang, Z. and X. Zhang, Argonautes compete for miR165/166 to regulate shoot apical meristem development. Curr Opin Plant Biol, 2012. 15(6): p. 652-8.

36. Kim, Y.S., et al., HD-ZIP III activity is modulated by competitive inhibitors via a feedback loop in Arabidopsis shoot apical meristem development. Plant Cell, 2008. 20(4): p. 920-933.

37. Liu, J., et al., MicroRNA319-regulated TCPs interact with FBHs and PFT1 to activate CO transcription and control flowering time in Arabidopsis. PLoS Genet, 2017. 13(5): p. e1006833.

38. Schommer, C., et al., Control of jasmonate biosynthesis and senescence by miR319 targets. PLoS Biol, 2008. 6(9): p. e230.

39. Lin, Y.F., et al., Genome-wide identification and characterization of TCP genes involved in ovule development of Phalaenopsis equestris. J Exp Bot, 2016. 67(17): p. 5051-66.

40. Martin-Trillo, M. and P. Cubas, TCP genes: a family snapshot ten years later. Trends Plant Sci, 2010. 15(1): p. 31-9.

41. Danisman, S., et al., Arabidopsis class I and class II TCP transcription factors regulate jasmonic acid metabolism and leaf development antagonistically. Plant Physiol, 2012. 159(4): p. 1511-23.

42. Tsuzuki, M. and Y. Watanabe, Profiling New Small RNA Sequences. Methods Mol Biol, 2017. 1456: p. 177-188.

43. Weigel, D. and J.L. Dangl, 10 years of Current Opinion in Plant Biology 1997-2007. Curr Opin Plant Biol, 2007. 10(6): p. 543-5.

44. Misteli, T. and G. Warren, 25 years of Current Opinion in Cell Biology. Curr Opin Cell Biol, 2013. 25(1): p. 1-2.

45. Nature Reviews Genetics. From the editors. Nat Rev Genet, 2011. 12(8): p. 517.

46. Saplaoura, E. and F. Kragler, Mobile Transcripts and Intercellular Communication in Plants. Enzymes, 2016. 40: p. 1-29.

47. Mostaan, S., et al., A novel combined method for cost-benefit production of DNA ladders. Adv Biomed Res, 2015. 4: p. 15.

48. Bastaki, N.K. and C.A. Cullis, Floral-dip transformation of flax (Linum usitatissimum) to generate transgenic progenies with a high transformation rate. J Vis Exp, 2014(94).

49. Haley, B., G. Tang, and P.D. Zamore, In vitro analysis of RNA interference in Drosophila melanogaster. Methods, 2003. 30(4): p. 330-6.

50. Livak, K.J. and T.D. Schmittgen, Analysis of relative gene expression data using real-time quantitative PCR and the 2(T)(-Delta Delta C) method. Methods, 2001. 25(4): p. 402-408. 
51. Horning, D.P. and G.F. Joyce, Amplification of RNA by an RNA polymerase ribozyme. Proc Natl Acad Sci U S A, 2016. 113(35): p. 9786-91.

52. Tang, G. and P.D. Zamore, Biochemical dissection of RNA silencing in plants. Methods Mol Biol, 2004. 257: p. 223-44.

53. Ghildiyal, M., et al., Endogenous siRNAs derived from transposons and mRNAs in Drosophila somatic cells. Science, 2008. 320(5879): p. 1077-81.

54. Mallory, A.C., et al., MicroRNA control of PHABULOSA in leaf development: importance of pairing to the microRNA 5' region. EMBO J, 2004. 23(16): p. 3356-64.

55. Zhong, R. and Z.H. Ye, Regulation of HD-ZIP III Genes by MicroRNA 165. Plant Signal Behav, 2007. 2(5): p. 351-3.

56. Ji, L.J., et al., ARGONAUTE10 and ARGONAUTE1 Regulate the Termination of Floral Stem Cells through Two MicroRNAs in Arabidopsis. Plos Genetics, 2011. 7(3).

57. Todesco, M., et al., A collection of target mimics for comprehensive analysis of microRNA function in Arabidopsis thaliana. PLoS Genet, 2010. 6(7): p. e1001031.

58. Wu, G. and R.S. Poethig, Temporal regulation of shoot development in Arabidopsis thaliana by miR156 and its target SPL3. Development, 2006. 133(18): p. 3539-47.

59. Luo, M., et al., [The role of miR319 in plant development regulation]. Yi Chuan, 2011. 33(11): p. 1203-11.

60. Schommer, C., et al., Repression of Cell Proliferation by miR319-Regulated TCP4. Molecular Plant, 2014. 7(10): p. 1533-1544.

61. Ramachandran, V. and X. Chen, Degradation of microRNAs by a family of exoribonucleases in Arabidopsis. Science, 2008. 321(5895): p. 1490-2.

62. Mohan, R., et al., Differentially Expressed MicroRNA-483 Confers Distinct Functions in Pancreatic beta- and alpha-Cells. J Biol Chem, 2015. 290(32): p. 19955-66.

63. Smith, J.E. and K.E. Baker, Nonsense-mediated RNA decay - a switch and dial for regulating gene expression. Bioessays, 2015. 37(6): p. 612-623.

64. Luna-Vital, D., et al., Anthocyanin condensed forms do not affect color or chemical stability of purple corn pericarp extracts stored under different pHs. Food Chem, 2017. 232: p. 639-647.

65. Wasila, H., et al., Peel effects on phenolic composition, antioxidant activity, and making of pomegranate juice and wine. J Food Sci, 2013. 78(8): p. C1166-72.

66. He, J., et al., Oxidative formation and structural characterisation of new alpha-pyranone (lactone) compounds of non-oxonium nature originated from fruit anthocyanins. Food Chem, 2011. 127(3): p. 984-92.

67. Hanhineva, K., et al., Impact of Dietary Polyphenols on Carbohydrate Metabolism. International Journal of Molecular Sciences, 2010. 11(4): p. 1365-1402.

68. Kennedy, D.O. and E.L. Wightman, Herbal Extracts and Phytochemicals: Plant Secondary Metabolites and the Enhancement of Human Brain Function. Advances in Nutrition, 2011. 2(1): p. 32-50.

69. Buer, C.S., N. Imin, and M.A. Djordjevic, Flavonoids: new roles for old molecules. J Integr 
Plant Biol, 2010. 52(1): p. 98-111.

70. Carlsen, M.H., et al., The total antioxidant content of more than 3100 foods, beverages, spices, herbs and supplements used worldwide. Nutrition Journal, 2010. 9.

71. Ferreyra, M.L.F., S.P. Rius, and P. Casati, Flavonoids: biosynthesis, biological functions, and biotechnological applications. Frontiers in Plant Science, 2012. 3.

72. Shi, M.Z. and D.Y. Xie, Biosynthesis and metabolic engineering of anthocyanins in Arabidopsis thaliana. Recent Pat Biotechnol, 2014. 8(1): p. 47-60.

73. Jurikova, T., et al., Black Crowberry (Empetrum nigrum L.) Flavonoids and Their Health Promoting Activity. Molecules, 2016. 21(12).

74. Aizza, L.C. and M.C. Dornelas, A genomic approach to study anthocyanin synthesis and flower pigmentation in passionflowers. J Nucleic Acids, 2011. 2011: p. 371517.

75. Shoeva, O.Y., et al., Regulation of the Flavonoid Biosynthesis Pathway Genes in Purple and Black Grains of Hordeum vulgare. PLoS One, 2016. 11(10): p. e0163782.

76. Suzuki, K., et al., RNA-seq-based evaluation of bicolor tepal pigmentation in Asiatic hybrid lilies (Lilium spp.). BMC Genomics, 2016. 17(1): p. 611.

77. Cavallini, E., et al., Functional diversification of grapevine MYB5a and MYB5b in the control of flavonoid biosynthesis in a petunia anthocyanin regulatory mutant. Plant Cell Physiol, 2014. 55(3): p. 517-34.

78. Berenschot, A.S. and V. Quecini, A reverse genetics approach identifies novel mutants in light responses and anthocyanin metabolism in petunia. Physiol Mol Biol Plants, 2014. 20(1): p. 1-13.

79. Guo, N., et al., Anthocyanin biosynthetic genes in Brassica rapa. BMC Genomics, 2014. 15 : p. 426.

80. Albert, N.W., et al., Light-induced vegetative anthocyanin pigmentation in Petunia. J Exp Bot, 2009. 60(7): p. 2191-202.

81. Wan, S., et al., PtrMYB57 contributes to the negative regulation of anthocyanin and proanthocyanidin biosynthesis in poplar. Plant Cell Rep, 2017.

82. Patra, B., et al., Transcriptional regulation of secondary metabolite biosynthesis in plants. Biochimica Et Biophysica Acta-Gene Regulatory Mechanisms, 2013. 1829(11): p. 1236-1247.

83. Xu, W.J., C. Dubos, and L. Lepiniec, Transcriptional control of flavonoid biosynthesis by MYB-bHLH-WDR complexes. Trends in Plant Science, 2015. 20(3): p. 176-185.

84. Qi, T., et al., The Jasmonate-ZIM-domain proteins interact with the WD-Repeat/bHLH/MYB complexes to regulate Jasmonate-mediated anthocyanin accumulation and trichome initiation in Arabidopsis thaliana. Plant Cell, 2011. 23(5): p. 1795-814.

85. Luo, Q.J., et al., An autoregulatory feedback loop involving PAP1 and TAS4 in response to sugars in Arabidopsis. Plant Mol Biol, 2012. 80(1): p. 117-29.

86. Ghildiyal, M. and P.D. Zamore, Small silencing RNAs: an expanding universe. Nat Rev Genet, 2009. 10(2): p. 94-108. 
87. Matzke, M., et al., RNA-mediated chromatin-based silencing in plants. Curr Opin Cell Biol, 2009. 21(3): p. 367-76.

88. Gou, J.Y., et al., Negative regulation of anthocyanin biosynthesis in Arabidopsis by a miR156-targeted SPL transcription factor. Plant Cell, 2011. 23(4): p. 1512-22.

89. Jia, X., et al., Small tandem target mimic-mediated blockage of microRNA858 induces anthocyanin accumulation in tomato. Planta, 2015. 242(1): p. 283-93.

90. Clough, S.J. and A.F. Bent, Floral dip: a simplified method for Agrobacterium-mediated transformation of Arabidopsis thaliana. Plant J, 1998. 16(6): p. 735-43.

91. Girardi, C., et al., Analysis of miRNA and mRNA expression profiles highlights alterations in ionizing radiation response of human lymphocytes under modeled microgravity. PLoS One, 2012. 7(2): p. e31293.

92. Wang, W.X., et al., The expression of microRNA miR-107 decreases early in Alzheimer's disease and may accelerate disease progression through regulation of beta-site amyloid precursor protein-cleaving enzyme 1. J Neurosci, 2008. 28(5): p. 1213-23.

93. Qiao, M.M., <FUNCTIONAL STUDIES OF HIGHLY EXPRESSED MICRORNAS IN ARABIDOPSIS T.pdf>. Master's Thesis, Michigan Technological University,, 2015.

94. Samad, A.F.A., et al., MicroRNA and Transcription Factor: Key Players in Plant Regulatory Network. Front Plant Sci, 2017. 8: p. 565.

95. Yeoh, G., S. Barton, and K. Kaestner, The International Journal of Biochemistry \& Cell Biology. Preface. Int J Biochem Cell Biol, 2011. 43(2): p. 172.

96. Ptashne, M., Regulation of transcription: from lambda to eukaryotes. Trends Biochem Sci, 2005. 30(6): p. 275-9.

97. Deaton, A.M. and A. Bird, $C p G$ islands and the regulation of transcription. Genes Dev, 2011. 25(10): p. 1010-22.

98. Barrett, L.W., S. Fletcher, and S.D. Wilton, Regulation of eukaryotic gene expression by the untranslated gene regions and other non-coding elements. Cell Mol Life Sci, 2012. 69(21): p. 3613-34.

99. Schlundt, A., et al., Structure-function analysis of the DNA-binding domain of a transmembrane transcriptional activator. Sci Rep, 2017. 7(1): p. 1051.

100. Liu, C.F., et al., Crystal structure of the DNA binding domain of the transcription factor T-bet suggests simultaneous recognition of distant genome sites. Proc Natl Acad Sci U S A, 2016. 113(43): p. E6572-E6581.

101. Jin, J.P., et al., PlantTFDB 3.0: a portal for the functional and evolutionary study of plant transcription factors. Nucleic Acids Research, 2014. 42(D1): p. D1182-D1187.

102. Ramachandran, P., A. Carlsbecker, and J.P. Etchells, Class III HD- ZIPs govern vascular cell fate: an HD view on patterning and differentiation. Journal of Experimental Botany, 2017. 68(1): p. 55-69.

103. Huijser, P. and M. Schmid, The control of developmental phase transitions in plants. Development, 2011. 138(19): p. 4117-29.

104. Usami, T., et al., The more and smaller cells mutants of Arabidopsis thaliana identify 
novel roles for SQUAMOSA PROMOTER BINDING PROTEIN-LIKE genes in the control of heteroblasty. Development, 2009. 136(6): p. 955-64.

105. Pulido, A. and P. Laufs, Co-ordination of developmental processes by small RNAs during leaf development. J Exp Bot, 2010. 61(5): p. 1277-91.

106. Miyashima, S., et al., Stem cell function during plant vascular development. EMBO J, 2013. 32(2): p. 178-93.

107. Elo, A., et al., Stem cell function during plant vascular development. Semin Cell Dev Biol, 2009. 20(9): p. 1097-106.

108. Ohashi-Ito, K., M. Matsukawa, and H. Fukuda, An atypical bHLH transcription factor regulates early xylem development downstream of auxin. Plant Cell Physiol, 2013. 54(3): p. 398-405.

109. Smith, Z.R. and J.A. Long, Control of Arabidopsis apical-basal embryo polarity by antagonistic transcription factors. Nature, 2010. 464(7287): p. 423-6.

110. Prigge, M.J., et al., Class III homeodomain-leucine zipper gene family members have overlapping, antagonistic, and distinct roles in Arabidopsis development. Plant Cell, 2005. 17(1): p. 61-76.

111. Rubio-Somoza, I. and D. Weigel, Coordination of Flower Maturation by a Regulatory Circuit of Three MicroRNAs. Plos Genetics, 2013. 9(3).

112. Lee, C. and S.E. Clark, A WUSCHEL-Independent Stem Cell Specification Pathway Is Repressed by PHB, PHV and CNA in Arabidopsis. Plos One, 2015. 10(5).

113. Schwarzkopf, M. and N.A. Pierce, Multiplexed miRNA northern blots via hybridization chain reaction. Nucleic Acids Res, 2016. 44(15): p. e129.

114. Rao, X., et al., An improvement of the $2^{\wedge}$ (-delta delta CT) method for quantitative real-time polymerase chain reaction data analysis. Biostat Bioinforma Biomath, 2013. 3(3): p. 71-85.

115. Eminaga, S., et al., Quantification of microRNA expression with next-generation sequencing. Curr Protoc Mol Biol, 2013. Chapter 4: p. Unit 417.

116. Song, A., et al., Transcriptome-Wide Survey and Expression Profile Analysis of Putative Chrysanthemum HD-Zip I and II Genes. Genes (Basel), 2016. 7(5).

117. Chen, X., et al., Genome-wide analysis of soybean HD-Zip gene family and expression profiling under salinity and drought treatments. PLoS One, 2014. 9(2): p. e87156.

118. Song, J.B., et al., Regulation of Leaf Morphology by MicroRNA394 and Its Target LEAF CURLING RESPONSIVENESS. Plant Cell Physiol, 2012.

119. Floyd, S.K., C.S. Zalewski, and J.L. Bowman, Evolution of class III homeodomain-leucine zipper genes in streptophytes. Genetics, 2006. 173(1): p. 373-388.

120. Zhu, Y.Y., et al., PtrHB7, a class III HD-Zip Gene, Plays a Critical Role in Regulation of Vascular Cambium Differentiation in Populus. Molecular Plant, 2013. 6(4): p. 1331-1343.

121. Itoh, J.I., et al., Developmental role and auxin responsiveness of class III homeodomain leucine zipper gene family members in rice. Plant Physiology, 2008. 147(4): p. 
1960-1975.

122. Zhang, Z.H. and X.R. Zhang, Argonautes compete for miR165/166 to regulate shoot apical meristem development. Current Opinion in Plant Biology, 2012. 15(6): p. 652-658.

123. Elhiti, M. and C. Stasolla, Structure and function of homodomain-leucine zipper (HD-Zip) proteins. Plant Signal Behav, 2009. 4(2): p. 86-8. 


\section{Appendix}

\section{The permission needed for including my published co-first}

\section{author research article as chapter 3 of my dissertation is shown}

below:

ASPB Member Center Contact Terms \& Privacy Plantae

$y$ in

\section{About $\mid$ ASPB Sections $\mid$ Membership $\mid$ Publications $\mid$ Meetings \& Events $\mid$ Awards \& Funding $\mid$ Education \& Outreach $\mid$ Advocacy $\mid$ News \& Updates Q}

Permission to Use Content from Plant Physiology® and The Plant Cell

Permission to make digital or hard copies of part or all of a work published in Plant Physiology or The Plant Cell is granted without fee for personal or classroom use, provided that copies are not made or distributed for profit or commercial advantage. Please include a citation in any standard format and a notice that the material is copyright by the American Society of Plant Biologists. If the use is commercial or if you wish to make multiple copies other than for educational purposes, please process your request through the Copyright Clearance Center at

Copyright Clearance Center Inc.

Re: Plant Physiology@/The Plant Cell

222 Rosewood Drive

Danvers, MA 01923 USA

Voice: $978-750-8400$

Fax: $978-750-4470$

Internet: http://www.copyright.com/

To Our Authors:

ASPB grants to authors whose work has been published in Plant Physiology@ or The Plant Cell the royalty-free right to reuse images, portions of an article, or full articles in any book, book chapter, or journal article of which the author is the author or editor. Reproductions must bear the full citation, the journal URL (www.plantphysio. org or www.plantcell.org), and the following notice: "Copyright American Society of Plant Biologists." ASPB further grants to authors the permission to make digital or hard copies of part or all of a work published in Plant Physiology@ or The Plant Cell without fee for personal or classroom use.
ASPB JOURNALS

ASPB Journals

OPENArticles

Plant Physiology

The Plant Cell

ASPB Top Authors

ASPB Policies \& Procedures

Librarian Resources

Permission to Reprint

Other ASPB Publications

The Arabidopsis Book

Biochemistry \& Molecular Biology of Plants (2nd edition)

Current Protocols in Plant Biology

Plant Direct

Plants Genes, Genomes and Genetics

Molecular Life of Plants

Teaching Tools in Plant Biology

My Life as a Plant

Online Image Library-BMBP textbook (Istedition)

ASPB News

ASPB Signal 